\title{
A Versatile Coexistence Decision-Making System for Efficient TV Whitespace Sharing among Whitespace Objects
}

\author{
M. Asif Raza, ${ }^{1}$ Zafar Iqbal, ${ }^{1}$ Sang-Seon Byun, ${ }^{2}$ Hyunduk Kang, ${ }^{3}$ and Heung-No Lee ${ }^{1}$ \\ ${ }^{1}$ School of Electrical Engineering and Computer Science, Gwangju Institute of Science and Technology, \\ Gwangju 500-712, Republic of Korea \\ ${ }^{2}$ Computer Engineering Department, Catholic University of Pusan, Busan 609-757, Republic of Korea \\ ${ }^{3}$ Electronics and Telecommunications Research Institute, 218 Gajeong-ro, Yuseong-gu, Daejeon 305-700, Republic of Korea
}

Correspondence should be addressed to Heung-No Lee; heungno@gist.ac.kr

Received 15 May 2017; Revised 14 August 2017; Accepted 24 August 2017; Published 22 October 2017

Academic Editor: Javier D. S. Lorente

Copyright (C) 2017 M. Asif Raza et al. This is an open access article distributed under the Creative Commons Attribution License, which permits unrestricted use, distribution, and reproduction in any medium, provided the original work is properly cited.

A coexistence decision-making (CDM) system for efficient TV whitespace (TVWS) sharing among whitespace objects (WSOs) is introduced in this paper. The proposed system is considered versatile in functionality as it jointly takes care of three distinct channel allocation features: (a) optimizing system quality of service (QoS) performance metrics, (b) improving TVWS utility, and (c) satisfying WSO channel demands. Regarding system QoS performance metrics, the TVWS sharing problem is defined as an optimization problem with an aim to maximize the system throughput and minimize unfairness in allocation. Supporting the WSOs channel demands in a TVWS sharing problem is a multifold task which requires elaborate consideration in different aspects of the system performance. To this end, the variations of the SNR of wireless frequency channels which result in variable throughput gain of the WSOs are also taken care of in the proposed CDM system. A fast channel allocation algorithm is then designed that implements the TVWS sharing mechanism in a reasonable amount of time. Additionally, the proposed algorithm improves the TVWS utility by promoting a novel frequency reuse method by exploiting the inter-WSO interference information. Simulation results show the superiority of the proposed algorithm over existing TVWS sharing algorithms.

\section{Introduction}

An unprecedented increase in the deployment of content delivery networks (CDNs) has resulted in the rapid growth of IP traffic. It is reported that, by the end of 2016, global IP traffic exceeded 1 zettabytes ( $10^{21}$ bytes) per year, of which $62 \%$ is attributed to CDNs [1]. It is also anticipated that by 2019 nearly two-thirds of global IP traffic will originate from non-PC devices, mainly portable and mobile devices [1]. On the other hand, currently available wireless spectrum is considered insufficient for accommodating such large volumes of data. Fortunately, the digitization of TV transmission has partially relinquished VHF and UHF spectrum [2]. Owing to its low loss and excellent propagation characteristics, the TV spectrum is considered a promising candidate for supporting the growing traffic over wireless channels. Considering the growing demand of the wireless spectrum, the regulatory bodies worldwide [3-5] have permitted unlicensed use of the TV spectrum under certain limits to protect the incumbents. However, the problem of coexistence of secondary devices operating in the same TV band was not dealt with by the regulatory bodies.

The coexistence among secondary devices operating in TV spectrum is considered a challenging task due to signal propagation characteristics of TV channels, spatiotemporal variation of TV spectrum, and disparity in network technologies of devices operating in the TV spectrum [6]. These diversities may cause coexistence issues, such as an unresolvable interference, spectrum congestion, and diversity in network size, as explained in [6-9]. To address coexistence issues and regulate access to TV spectrum, IEEE has proposed an 802.19.1 standard [10]. The standard provides a set of procedures to enable coexistence among secondary networks operating in heterogeneous network technologies in TVWS, namely, WSOs. 
A set of procedures that ensures peaceful coexistence among a set of WSOs operating in the same spectrum is referred to as CDM [11]. In this paper, we define an 802.19.1 compliant CDM system that performs TVWS sharing among a set of WSOs, operating in dissimilar MAC/PHY layer technologies and registered in the coexistence manager (CM); an entity in 802.19.1 coexistence system as shall be defined in Section 3.1. Note that the TVWS refers to the TV spectrum not in use by licensed operators in a spatiotemporal region [10]. The TVWS sharing problem is modeled as an optimization problem with an aim to maximize the system performance metrics like system throughput and fairness in TVWS allocation. The optimization problem is constrained that the channel demands of the WSOs registered in the neighboring CMs are satisfied. In this perspective, variations of the SNR of wireless frequency channels which result in variable throughput gain of the WSOs are taken care of. Note that the neighboring CMs refer to the set of CMs whose WSOs create interference to each other and such WSOs are neighboring WSOs. Thus, the proposed CDM system differs from the notion of traditional node-based, linkbased, or base-station based channel allocation as reported in the TVWS sharing literature. Moreover, the proposed system also improves the TVWS utility by implementing a frequency reuse (FR) method to spatially reuse the available TV spectrum in a joint time-frequency domain in an ad hoc coexisting environment. In this paper, the ad hoc coexisting environment refers to the coexistence of both infrastructure based WSOs like WLAN and ad hoc WSOs like personal area network. An ad hoc WSO accounts for a local area network that is built spontaneously, as devices connect with each other. The CDM system proposed in this paper is unique, to the best of our knowledge, in the sense that it jointly focuses on three distinct TVWS sharing objectives: (a) optimizing system performance metrics during TVWS sharing among WSOs registered in neighboring CMs in 802.19.1 system, (b) improving the TVWS utility by implementing the FR in a joint time-frequency domain, and (c) taking care of the channel demands of the heterogeneous WSOs. Such a joint focus to implement multiple distinct channel allocation features makes the proposed system a versatile CDM system.

The remainder of the paper is organized as follows. Section 2 reviews some related studies. Section 3 summarizes technical background required to establish the baseline for the techniques used in the paper. The system description and problem formulation are defined in Section 4. Section 5 discusses the solution method and the proposed algorithm. Section 6 presents the simulation results and compares the proposed algorithm with existing algorithms. Finally, Section 7 concludes the paper.

\section{Previous Work}

In this section, we summarize some standards and algorithms developed for achieving coexistence among secondary users.

IEEE 802.15.2 [12] and 802.15.4 [13] have partially addressed the coexistence issue among devices operating on wireless local area networks and low-power wireless personal area networks, respectively. However, these networks operate on industrial, scientific, and medical bands. On the other hand, IEEE 802.22 has recently defined PHY and MAC layer extensions for TVWS. Similarly, IEEE 802.11af [14] has adopted new cognitive radio features to protect incumbents and achieve efficient spectrum utilization among unlicensed devices. IEEE 802.22.1 has also defined methods for peaceful coexistence when a low-power licensed device such as a microphone broadcaster and an unlicensed device both coexist and share the same channel [15]. The European Computer Manufacturers Association (ECMA) has also defined a specification (ECMA 392) for personal/portable cognitive wireless networks operating in TVWS [16]. However, all these standards define self-coexistence in TVWS operations. Nonavailability of cross-platform coexistence mechanisms shall cause issues such as an inability to diagnose interference among networks with dissimilar network technologies and may lead to inefficient utilization of the scarce wireless spectrum [11]. Perceiving the need for cross-platform coexistence mechanisms, IEEE has defined an 802.19.1 standard. This standard provides coexistence protocols and policies for efficient utilization of TVWS across platforms [10].

On algorithmic perspective, a CDM algorithm that results in fair TVWS sharing among neighboring CMs is presented in [10]. The algorithm applies max-min fairness technique to establish fair share distribution during the TVWS sharing process. The issue with the algorithm in [10] is that it focuses on fairness in allocation while no consideration to effective utilization of the available TVWS is taken care. Considering the scarcity of the TV spectrum, especially in highly congested spectrum environments, the effective utilization of the available TV spectrum is also an important factor to be considered. Hessar and Roy [17] have discussed the TVWS sharing formulations in secondary cellular networks. The authors adopt heuristic based approaches to defining greedy algorithms to tackle the identified TVWS sharing problems. However, the proposed greedy algorithm for throughput maximization subproblem searches the entire network to find an optimal solution. For such an algorithm, search over the space of a possibly very large number of network and channel collocation combinations leads to a high runtime complexity to find an optimal solution. An algorithm for opportunistic whitespace sharing among secondary networks has been presented as a graph coloring problem in [18]. The channel sharing algorithm in [18] solves the sharing problem by classifying the sharing process as network wide channel sharing and its localized version. This scheme, however, has performance issue when interference among neighboring access points is relatively high. This situation is quite common in highly congested areas where many collocated WSOs are deployed. Bahrak and Park [11] proposed an algorithm for CDM among heterogeneous networks. The sharing problem in [11] is modeled as a weightedsum multiobjective optimization problem (MOOP) that is solved using a modified Boltzmann machine. However, an issue in the weighted-sum approach is that it does not find Pareto optimal points in nonconvex regions of the solution space boundary [19]. Thus, some of the potential Pareto optimal points are possibly missed by the weighted-sum method. 
Khalil et al. have also performed TVWS sharing among heterogeneous networks by defining an interference graph of the networks [20]. A two-stage algorithm is then designed to achieve spectrum sharing among graph nodes. The algorithm maximizes fairness by maximizing the frequency reuse. However, the channel sharing algorithm in [20] has polynomial runtime complexity $\mathcal{O}\left(N^{3}\right)$, for the number of networks $(N)$. This complexity shows that, in areas with a high number of deployed networks, the algorithm shall require substantial channel allocation time. Zhang et al. [21] adapt ecology based species competition model to develop a coexistence mechanism called ecological Species Competition based HEterogeneous networks coexistence MEchanism (SCHEME). The SCHEME enables each coexisting network to adjust achieved bandwidth per its QoS requirements dynamically. However, the SCHEME requires the number of channels to be larger than the number of coexisting networks. Such condition cannot be fulfilled in highly congested urban areas where a limited number of TV channels is available for unlicensed use. We have addressed this issue in the channel allocation mechanism defined in this paper.

On the other hand, some of the existing TVWS sharing algorithms have implemented the concept of FR. For example, in [22], Bian et al. have implemented the concept of FR in sharing a single TV channel among Cognitive Radios (CR). The CR networks operating in orthogonal frequency division multiple access apply the uplink soft FR concept [23]. Again, the proposed method is defined for CR systems deployed in cellular infrastructure. Similarly, Hessar and Roy [17] have presented an FR method in cellular networks operating in TVWS. Moreover, the algorithm proposed in [17] orthogonalizes WSOs in frequency domain only. None of the existing TVWS sharing algorithms reuses TVWS in a joint, time-frequency domain for WSOs operating in an ad hoc coexisting environment. Spectrum reuse in both time and frequency domains shall result in even a better utilization of the available TVWS, as discussed in Section 6.3.

Some genetic algorithms (GA), defined for implementing the channel sharing problem, also exist in the literature. For example, the authors in [24] use a GA-based reliability model to assign channels to mobile hosts based on the reliability of the base station and the channels to enhance the overall reliability of the mobile network system. The results show that this method requires higher number of iterations and generally higher number of available channels than the number of mobile hosts in order to achieve higher reliability. Similarly, Shrestha et al. propose a GA-based joint out-ofband spectrum sensing and channel allocation scheme for cognitive radio networks [25]. The joint sensing and resource allocation optimization problem has been formulated using fitness functions of sensing utility and the data transmission utility. Jiao and Joe consider a new cognitive radio network model with heterogeneous primary users operating simultaneously via multiradio access technology [26]. It focuses on energy efficient resource allocation and use a GA-based scheme to obtain an optimal solution in terms of power and bandwidth. The authors in [27] proposed solutions for the problem of efficient resource allocation (radio spectrum and power) in the OFDMA-based multicast wireless system

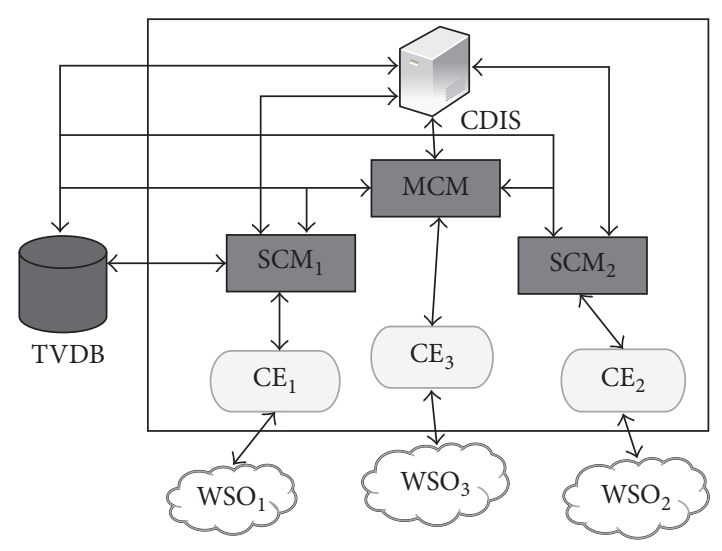

FIGURE 1: IEEE 802.19.1 TVWS system architecture. The TVWS database and WSOs interact with the 802.19.1 architecture externally.

that balances the trade-off between maximizing the total throughput and ensuring a flexible and controllable spectrum sharing among multicast groups. It proposes two separate optimization methods for subcarriers and power and a GAbased joint optimization scheme is used. Results show that the proposed schemes can attain a high total sum-rate and more flexible and fair distribution of the available bandwidth among multicast groups.

The GAs in [24-27] and such literature work [28, 29] are well suited for multiobjective optimization problems that require searching over a large space under several constraints. However, GA-based methods are computationally expensive and therefore not suitable for the optimization problem with single objective function and a small search space, like the one defined in this paper. Therefore, GA suffers from the drawbacks of slow convergence speed and low stability. The channel allocation in highly dynamic spectrum environments requires an algorithm that can do allocation process in a quick runtime. Therefore, rather than applying the GA method, the nonlinear, binary constrained optimization problem, defined in this paper, is transformed into linear optimization problem. Such formulation helps us to apply linear programming solvers to solve the optimization problem and complete the allocation process in a quick, linear runtime.

\section{Technical Terms and Research Focus}

3.1. Technical Terms. In this section, we define technical terms that form baseline of the proposed TVWS sharing system, defined in the next section. The proposed system is based on the coexistence system architecture as described in [10] and shown in Figure 1. The coexistence system in [10] has three logical components: coexistence manager (CM), coexistence enabler (CE), and a coexistence discovery and information server (CDIS).

(i) The CE registers a WSO to the CM and acts as a communication bridge by translating messages between the WSO and the CM serving the WSO. 
(ii) The CM makes coexistence decisions for WSOs registered in it. Moreover, it is required to interact with other CMs, called neighboring CMs in [10], to resolve coexistence issues among WSOs served by neighboring CMs. In general, it sends configuration commands and control information to the CE.

(iii) The CDIS provides coexistence discovery services like coexistence set information to CMs for registered WSOs.

(iv) The TVWS database (TVDB), as shown in Figure 1, is not part of the coexistence system architecture. It contains information about channels available in the geographic region of each WSO registered with the 802.19.1 system. The TVWS database provides information about the set of TV channels free for whitespace activity to the CMs.

A WSO may register with the IEEE 802.19.1 system before operating in the TV spectrum. In the registration process, a general principle for a WSO to acquire a TV channel is defined in IEEE 802.19.1, summarized as follows. A WSO may perform spectrum sensing to identify and select an available free TV channel or alternatively, it may send a channel allocation request to its serving CM. If no free channel is available in the geographic region of the WSO, the CM may perform channel sharing among the requesting WSO and the WSOs preallocated a TV channel. If such WSOs are registered with other CMs, the CM serving the channel requesting WSO interacts with the other CMs to perform channel sharing. These CMs are called neighboring CMs to the requesting $\mathrm{CM}$. In this channel sharing procedure, two types of topologies are defined in the 802.19.1 [10]. A distributed CDM topology where neighboring CMs mutually interact to perform channel sharing among WSOs registered within them. A centralized CDM topology where multiple CMs agree to select one of them a master CM (MCM) and rest of the CMs become slave CM (SCM) [10], as shown in Figure 1. Each SCM provides essential information about operating parameters, including the channel characteristics of each WSO registered within it and its channel demands to the MCM. The MCM performs coexistence services like radio resource allocation to WSOs registered in the SCMs. Some other terms used in the paper are defined as follows.

(i) A WSO is an entity in 802.19 .1 system that represents a TVWS device or network of devices.

(ii) The channel occupancy is the duty cycle in a percentage that a network (WSO) occupies a channel [10].

(iii) The window time is a slot duration of a scheduling repetition period that satisfies the essential system QoS performance [10].

(iv) The coexistence set (CS) of a $w$ th WSO is a set of WSOs that are registered in the neighboring CMs that may affect the performance of the $w$ th WSO. In other words, it is a set of WSOs which create interference to the $w$ th WSO.
3.2. Research Focus. The TVWS sharing problem is defined as follows.

Given a set of available TV channels, a set of CMs with each CM having at least one WSO registered in it and WSOs channel demands share the TV channels among WSOs such that the following objectives are achieved.

(1) Maximize the system throughput.

(2) Minimize unfairness in allocation among WSOs registered in neighboring CMs.

(3) Fulfill desired channel demands of the allocated WSOs.

These objectives contradict each other. For example, maximizing the system throughput shall decrease fairness in allocation. Note that, from a spectrum allocation perspective, fairness is regarded as equity in access to the resource, the TV spectrum. In other words, being free to use, each network should have an equal opportunity to an access to the given TV spectrum.

Similarly, fulfilling the second and third objectives in conjunction, under the scarcity of the available TVWS, restricts the system accommodating as many as WSOs in the TVWS. Thus, maximizing the fairness while satisfying the channel demands of each allocated WSO is quite complicated in highly congested spectrum environments [30]. Therefore, the fairness in allocation is measured at CM level. The fairness among CMs is deemed at minimum if at least a single WSO in each CM gets the channel.

Considering the above conditions, we design a CDM system, as will be defined in Section 4.1. The system is designed to implement at the MCM in the centralized topology in 802.19.1, as shown in Figure 1. The system makes use of the information from information messages defined in the 802.19.1 [10] to apply various procedures for defining the proposed TVWS sharing problem as an optimization problem. For example, the WSO registration clause in [10] defines different information acquiring messages that permit a CM to collect desired channel demands, channel statistics, coexistence set elements, available TV channels, and related information from WSOs registered within it or with neighboring CMs. Moreover, the inter-CM information sharing messages are also defined in [10]. We assume that using such message templates, the neighboring CMs exchange respective WSOs information with MCM. In order to solve the TVWS sharing problem, the CDM system in MCM then implements a channel allocation process, as will be defined in Section 5.3. The algorithm defined in Section 5.3 makes use of such information available at MCM to implement the subgradient method to solve the TVWS sharing dual problem, Section 5.2, to identify a set of WSOs to allocate the TV channels.

The channel allocation process also implements a novel spectrum reuse method, as shall be discussed in Section 5.5, to have an efficient use of the available TVWS. The spectrum reuse step is also made in compliant with the 802.19 .1 by repeated channel allocation using an interference matrix. The CDM defines the interference matrix using the WSOs' CS information available at MCM, as shall be discussed in Section 5.4. Note that the CS information is provided by 
TABLE 1: Defined parameters.

\begin{tabular}{|c|c|c|}
\hline Symbol & Description & Value \\
\hline \multicolumn{3}{|c|}{ Input variables } \\
\hline $\mathscr{C}$ & A set of $C$ CMs in the system & $\mathscr{C}=\{1,2, \ldots, C\}$ \\
\hline $\mathscr{W}^{c}$ & A set of NID of $W$ WSOs registered in the $c$ th CM & $\mathscr{W}^{c}=\left\{\mathrm{NID}_{1}, \mathrm{NID}_{2}, \ldots, \mathrm{NID}_{W}\right\}$ \\
\hline $\mathscr{J}$ & A set of permissible TV channels in the system & $\mathscr{J}=\{1,2, \ldots, J\}$ \\
\hline $\mathscr{D}$ & Channel demands of WSOs, as defined in the system in (1) & - \\
\hline$O_{w, j}^{c}$ & COT that translates desired occupancy demand of $w$ th WSO on a $j$ th channel & $\mathbf{O}_{w}^{c}=\left[O_{w, j}^{c}\right]_{1 \times J}, O_{w, j}^{c} \in \mathbb{R}_{\left[0, T_{j}\right]}$ \\
\hline$I_{w, m}(j)$ & Indicator variable encoding $m$ th WSO interference to $w$ th WSO on a $j$ th channel & $I_{w, m}(j):= \begin{cases}1 & \text { if } m \text { interfers } w \\
0 & \text { otherwise }\end{cases}$ \\
\hline $\mathcal{S}_{w, j}$ & $\begin{array}{l}\text { Set of WSOs } m \in \mathscr{W} \text { such that } m \text { th WSO transmission interferes with } w \text { th WSO } \\
\text { transmission on } j \text { th channel }\end{array}$ & $\mathcal{S}_{w, j}=\{m \in \mathscr{W}\}$ \\
\hline$y_{w, j}$ & A variable indicating whether $m$ th WSO interferes with $w$ th WSO on the $j$ th channel? & $y_{w, j}:= \begin{cases}1 & \exists w \in \mathscr{W}: m \in \mathcal{S}_{w, j} \\
0 & \text { else }\end{cases}$ \\
\hline$z_{w, j}^{c}$ & An element of the matrix $\mathbf{Z}$ defining accessibility of $j$ th channel to $w$ th WSO & $\begin{cases}1 & \text { if } j \text { accessible to } w \text { th WSO } \\
0 & \text { else }\end{cases}$ \\
\hline \multicolumn{3}{|c|}{ Output variables } \\
\hline$x_{w, j}^{c}$ & Element of matrix $\mathbf{X}$ defining allocation status of $w$ th WSO on $j$ th channel & $x_{w, j}^{c}:= \begin{cases}1 & \text { if channel allocated } \\
0 & \text { otherwise }\end{cases}$ \\
\hline
\end{tabular}

the coexistence discovery algorithm as defined in [10]. The channel allocation process is then executed repeatedly to spatially reuse the TV spectrum to the unallocated WSOs that should not cause interference to preallocated WSOs. The proposed channel allocation solution is thus made smoothly integrable to the 802.19 .1 system.

\section{System Description and Problem Formulation}

In the following section, a centralized CDM system is designed that implements a channel allocation process, as shall be discussed in Section 5, to implement the TVWS sharing problem defined in Section 3.2.

\subsection{System Model. The CDM system is defined as follows:}

$$
\mathbf{X}=\operatorname{TVWS}(\mathscr{C}, \mathscr{J}, \mathscr{Z}, \mathscr{T}, \mathscr{D}) \text {. }
$$

The system parameters are defined as follows. Let $c$ be an index to a set of $C$ neighboring CMs in the system, denoted as $\mathscr{C}$ in Table 1. Let $\mathscr{W}^{c}, \forall c \in \mathscr{C}$ be a set of network IDs of WSOs registered in the $c$ th $\mathrm{CM}$, as shown in Table 1 . Let the $w$ th WSO be registered in the cth CM. Let $\mathrm{NID}_{w} \in \mathscr{W}^{c}$ represent an identifier of the network that the wth WSO represents. Note that the identifier of a network represents an entity in the network that interacts with the external world, like the CM in this paper. The entity varies from network type to network type. For example, in the case of IEEE 802.11 type WSO, the NID contains the basic service set identifier used by the WSO.

Let $j$ be an index to the set of all permissible TV whitespace channels, $\mathscr{J}=\{1,2, \ldots, J\}$, where each set element corresponds to a TV channel number, defined on the basis of the regulatory authority rulings. For example, in USA where FCC defines each TV channel to be $6 \mathrm{MHz}$ bandwidth in V/UHF band, therefore, $\mathscr{J}=\{2,3, \ldots, 36,38, \ldots, 51\}$ in the USA. The availability of a TV channel to a $w$ th WSO is a function of geographic location of the WSO and the primary user activity in the region. Therefore, the availability of a TV channel for the secondary use varies spatiotemporally and needs to be determined. We assume that a channel sensing mechanism, as defined in [10], is implemented such that the TVDB contains the set of TV whitespace channels available in the geographic region of each WSO registered in the CMs in the system. Let $j$ be an index to the set $\mathscr{F}$; then, $j$ th channel availability status to the $w$ th WSO, registered in $c$ th CM, is represented by an indicator function defined as

$$
\begin{aligned}
& z_{w, j}^{c} \\
& := \begin{cases}1, & \text { if } j \text { th channel in } \mathscr{J} \text { is available to } w \text { th WSO } \\
0, & \text { otherwise. }\end{cases}
\end{aligned}
$$

The availability of $J$ channels to the $w$ th WSO, registered in cth CM, is thus represented by a vector of indicator functions defined as

$$
\mathbf{z}_{w}^{c}=\left(z_{w, 1}^{c}, \ldots, z_{w, J}^{c}\right)
$$

The set of channels available to $W$ WSOs registered in $c$ th CM is defined as

$$
\mathbf{Z}^{c}=\left(\mathbf{z}_{1}^{c}, \mathbf{z}_{2}^{c}, \ldots, \mathbf{z}_{W}^{c}\right)^{\mathrm{T}}, \quad \forall c \in \mathscr{C} .
$$




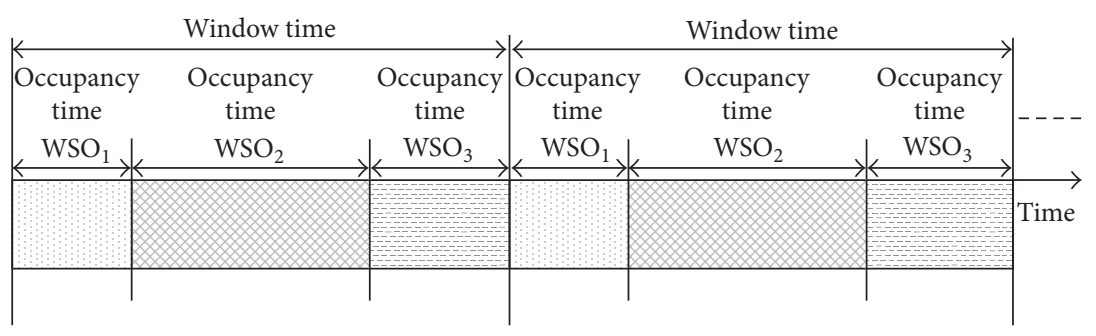

FIGURE 2: Scheduling transmission periods for three WSOs on a TV channel.

The system parameter $\mathscr{Z}$ is then defined as follows:

$$
\mathscr{Z}=\left\{\mathbf{Z}^{1}, \mathbf{Z}^{2}, \ldots, \mathbf{Z}^{C}\right\} \text {. }
$$

The parameter $\mathscr{T}$ in the system in (1) represents the set of window times for the channels in the set $\mathscr{J}$. In 802.19.1, an algorithm is provided that enables CMs to define the slot duration of the window time. We assume the CMs implement such an algorithm to define the window time, $T_{j}, \forall j \in \mathscr{J}$, which is then used to define system parameter as

$$
\mathscr{T}=\left\{T_{1}, \ldots, T_{J}\right\}
$$

The system parameter $\mathscr{D}$ in (1) encodes channel demands of CMs, defined as follows. In 802.19.1 [10], a Discovery Information abstraction is provided that allows WSOs to send channel statistics and channel demands like SINR, desired channel occupancy, desired bandwidth, and so forth, to their serving CM [10]. Such information of heterogeneous WSOs is used to define a set of channel demands of $w$ th WSO as follows.

Let $\operatorname{SINR}_{w, j}^{c}$ represent the quality of $j$ th channels to $w$ th WSOs registered in $c$ th CM. The channel quality is measured in terms of signal to interference and noise ratio (SINR) which depends on interference from primary-to-secondary users and noise floor due to environmental factors. We assume that an interference discovery mechanism is in place that enables each WSO to measure SINR value on each of the channels in $\mathscr{F}$, as will be further discussed in Section 5.4. The quality of all $J$ channels to $w$ th WSO is then defined as

$$
\mathbf{s}_{w}^{\prime c}=\left(\operatorname{SINR}_{w, 1}^{c}, \operatorname{SINR}_{w, 2}^{c}, \ldots, \operatorname{SINR}_{w, J}^{c}\right), \quad \forall w \in \mathscr{W}^{c} .
$$

Let $p_{w, j}^{c}$ be the allowed transmission power to $w$ th WSO in the $j$ th channel. The allowed transmission power to $w$ th WSO on $J$ channels is then defined as

$$
\mathbf{p}_{w}^{\prime c}=\left(p_{w, 1}^{c}, \ldots, p_{w, J}^{c}\right), \quad \forall w \in \mathscr{W}^{c}
$$

Let $B_{w}^{c}$ be the bandwidth demand of $w$ th WSO. The number of channels required by $w$ th WSO is then calculated as

$$
n_{w}^{c}=\frac{B_{w}^{c}}{b_{j}}, \quad \forall w \in \mathscr{W}^{c}, \forall c \in \mathscr{C},
$$

where $b_{j}$ represents the channel bandwidth. Let $O_{w, j}^{c}$ translate to a timeslot, here called channel occupancy time (COT) in a window time, such that the $w$ th WSO registered in $c$ th CM can achieve its desired channel occupancy in the allocated $j$ th channel. The relation of COT to a channel window time is shown in Figure 2, where three WSOs are scheduled in the window time in a single TV channel. The COTs of $w$ th WSO in $J \mathrm{TV}$ channels are then represented as

$$
\mathbf{o}_{w}^{\prime c}=\left(O_{w, 1}^{c}, \ldots, O_{w, J}^{c}\right)
$$

The channel demand set of $w$ th WSO is then defined as follows:

$$
\left\{\mathbf{s}_{w}^{\prime c}, \mathbf{p}_{w}^{\prime c}, n_{w}^{c}, \mathbf{o}_{w}^{\prime c}\right\}, \quad \forall c \in \mathscr{C}, \forall w \in \mathscr{W}^{c}
$$

The channel demand set of $c$ th CM is then defined using channel demands of its registered WSOs as follows:

$$
\mathscr{D}^{\prime c}=\left\{\mathbf{s}^{c}, \mathbf{p}^{c}, N^{c}, \mathbf{o}^{c}\right\}, \quad \forall c \in \mathscr{C},
$$

where $\mathbf{s}^{c}=\left(\mathbf{s}_{1}^{\prime c}, \ldots, \mathbf{s}_{W}^{\prime c}\right), \mathbf{p}^{c}=\left(\mathbf{p}_{1}^{\prime c}, \ldots, \mathbf{p}_{W}^{\prime c}\right), N^{c}=\left(n_{1}^{c}, \ldots\right.$, $\left.n_{W}^{c}\right)$, and $\mathbf{o}^{c}=\left(\mathbf{o}_{1}^{\prime c}, \ldots, \mathbf{o}_{W}^{\prime c}\right)$. Let $\mathbf{S}=\left(\mathbf{s}^{1}, \mathbf{s}^{2}, \ldots, \mathbf{s}^{C}\right)^{\mathrm{T}}$, $\mathbf{P}=\left(\mathbf{p}^{1}, \mathbf{p}^{2}, \ldots, \mathbf{p}^{C}\right)^{\mathrm{T}}, \mathbf{N}=\left(N^{1}, N^{2}, \ldots, N^{C}\right)^{\mathrm{T}}$, and $\mathbf{O}=$ $\left(\mathbf{o}^{1}, \mathbf{o}^{2}, \ldots, \mathbf{o}^{\mathrm{C}}\right)^{\mathrm{T}}$; the system parameter $\mathscr{D}$ is then defined using the channel demands of all neighboring CMs as follows:

$$
\mathscr{D}=\{\mathbf{S}, \mathbf{P}, \mathbf{N}, \mathbf{O}\} .
$$

The system in (1) then executes the channel allocation algorithm, as will be discussed in Section 5, to allocate TV channels to the WSOs registered in the neighboring CMs such that the allocation satisfies the required system QoS performance. The system QoS performance is preserved if the following allocation condition is satisfied:

$$
\sum_{c \in \mathscr{C}} \sum_{w \in \mathscr{W}^{c}} O_{w, j}^{c} \leq T_{j}, \quad \forall j \in \mathscr{J},
$$

where $T_{j}$ refers to the window time in a $j$ th channel. The algorithm proposed in Section 5 solves the TVWS sharing optimization problem, as will be defined in (21a)-(21e), and outputs a channel allocation matrix $\mathbf{X}$, defined as follows. Let $x_{w, j}^{c} \in\{0,1\}$ be a binary decision variable such that if $x_{w, j}^{c}=1$, the $j$ th channel is allocated to the $w$ th WSO registered in cth CM; otherwise, $x_{w, j}^{c}=0$. The allocation status of WSOs 
registered in the neighboring CMs is then represented by a matrix $\mathbf{X}$ as

$$
\mathbf{X}:=\left[\begin{array}{cccc}
x_{1,1}^{1} & x_{1,2}^{1} & \cdots & x_{1, J}^{1} \\
& & \vdots & \\
x_{W^{1}, 1}^{1} & x_{W^{1}, 2}^{1} & \cdots & x_{W^{1}, J}^{1} \\
x_{1,1}^{2} & x_{1,2}^{2} & \cdots & x_{1, J}^{2} \\
& & \vdots & \\
x_{W^{c}, 1}^{c} & x_{W^{c}, 2}^{c} & \vdots & x_{W^{c}, J}^{c} \\
& & \vdots & \\
x_{W^{C}, 1}^{C} & x_{W^{C}, 2}^{C} & \cdots & x_{W^{C}, J}^{C}
\end{array}\right],
$$

where $W^{c}=\left|\mathscr{W}^{c}\right|, \forall c \in \mathscr{C}$, that is, the number of WSOs registered in the $c$ th $\mathrm{CM}$. The $w$ th row in the $\mathbf{X}$ represents the channels allocation status, in the set $\mathscr{J}$, to the $w$ th WSO registered in $c$ th $\mathrm{CM}$. The $j$ th column in the $\mathbf{X}$ represents the channels allocation status of all the WSOs, from all the CMs in the set $\mathscr{C}$. The allocation matrix $\mathbf{X}$ thus orthogonalizes WSOs, registered in the neighboring CMs, in a joint frequencytime domain. The WSOs scheduled on different channels can transmit at the same time using their respective allotted channel (frequency slot), while WSOs scheduled on the same channel can transmit in their respective time slot (here COT).

The system in (1) thus implements the TVWS sharing problem, defined in Section 3.2, as an optimization problem, as discussed in the following section.

4.2. Problem Formulation. In this section, the proposed TVWS sharing problem is formulated as an optimization problem using well-established proportional fairness method. It is because the proportional fairness is considered one of the most suitable methods to achieve a trade-off between two competing interests [31-33]. Originally, Kelly defined the proportional fairness as an adjustment process which adjusts the rates of users according to the charges they pay. The proportional fairness method thus was defined for elastic traffic in computer network services [34]. Similarly, in the channel sharing literature, a proportionally fair allocation mostly has been achieved by adjusting the rates of the users based upon some performance criteria like maximizing the resource utilization and so forth $[35,36]$. However, applying the proportional fairness in its original form to model the TVWS sharing problem proposed in this paper is not suitable. It is because the third objective in the problem defined in Section 3.2 makes the resource allocation as binary decision allocation; that is, a channel is either allocated to a WSO, $x_{w, j}^{c}=1$, or not, $x_{w, j}^{c}=0$. Therefore, WSO allocation (here COT) adjustment is not possible. Consequently, we rewrite the proportional fairness in a binary decision allocation perspective as follows.

Let the maximum data rate that the $w$ th WSO can achieve on $j$ th channel be defined by using Shannon channel capacity formula,

$$
r_{w, j}^{c}=b_{j} \log \left(1+\operatorname{SINR}_{w, j}^{c}\right) .
$$

The maximum rate $r_{w, j}^{c}, \forall w \in \mathscr{W}^{c}$, is then used to define a utility function as a normalized rate achieved by $c$ th CM in $j$ th channel as follows:

$$
\mathcal{u}_{c, j}=\sum_{w \in \mathscr{W}^{c}} \frac{x_{w, j}^{c} r_{w, j}^{c}}{O_{w, j}^{c}+\delta_{O_{w, j}^{c} 0}},
$$

where $\delta_{\mathrm{O}_{w, j}^{c} 0}$ defines Kronecker delta function as

$$
\delta_{O_{w, j}^{c} 0}:= \begin{cases}1, & \text { if } O_{w, j}^{c}=0 \\ 0, & \text { otherwise. }\end{cases}
$$

This function prevents denominator term in (17) from becoming zero. The utility function in (17) measures the worth of the resource (channel) to $c$ th $\mathrm{CM}$; that is, given a channel is allocated to the WSOs in the $c$ th CM for the duration of $\sum_{w \in \mathscr{W}^{c}} O_{w, j}^{c}$, how does it translate for the $\mathrm{CM}$ in terms of the achieved throughput. In other words, maximizing the function in (17) shall prefer a CM with WSOs achieving high data rate and lower channel occupancy demand over a CM with WSOs achieving low data rate and high channel occupancy demand. Such preference based allocation shall lead to an efficient use of the resources (TVWS). The distribution $\mathbf{U}=\left[\mathcal{U}_{c, j}\right]_{C \times J}$ is then said to be proportionally fair if it is feasible and for all other feasible solutions $\mathbf{V}=\left[v_{c, j}\right]_{C \times J}$, the following holds [34]:

$$
\sum_{c \in \mathscr{C}} \sum_{j \in \mathcal{F}} \frac{v_{c, j}-\mathscr{U}_{c, j}}{\mathcal{U}_{c, j}} \leq 0 .
$$

It has been shown in $[34,37]$ that the rates achieved by users become proportionally fair if the sum of logarithmic rates obtained is optimized. Moreover, it is shown in [38] that if all rates are proportionally fair, they maximize the throughput over all other feasible throughputs. Therefore, if the logarithmic sum of the utility function in (17) is maximized, the normalized rate achieved by neighboring CMs shall become proportionally fair. A $j$ th channel is said to be allocated to the $c$ th CM if at least one of its registered WSOs is scheduled on the channel. The allocation status of the channels in the $\mathscr{J}$, to the $c$ th CM, is then defined as follows:

$$
\mathbf{x}^{c}:=\left[\begin{array}{cccc}
x_{1,1}^{c} & x_{1,2}^{c} & \cdots & x_{1, J}^{c} \\
& & \vdots & \\
x_{W^{c}, 1}^{c} & x_{W^{c}, 2}^{c} & \cdots & x_{W^{c}, J}^{c}
\end{array}\right] .
$$

Let $\mathbf{1}=(1,1, \ldots, 1)_{1 \times J}$. Let $\mathbf{O}_{j} \in \mathbf{O}$ be the $j$ th column vector in COT demand matrix in the system parameter $\mathscr{D}$, defined as $\mathbf{O}_{j}=\left(O_{1, j}^{1}, O_{2, j}^{1}, \ldots, O_{W^{1}, j}^{1}, O_{1, j}^{2}, \ldots, O_{W^{C}, j}^{C}\right)^{\mathrm{T}}$, where $W^{c}=$ $\left|\mathscr{W}^{c}\right|, \forall c \in \mathscr{C}$. Let $\mathbf{X}_{j} \in \mathbf{X}$ represent the $j$ th column vector of the allocation matrix $\mathbf{X}$. The TVWS sharing problem is then defined as follows:

$$
\max \sum_{c \in \mathscr{C}} \sum_{j \in \mathscr{J}} \log \left(\mathscr{U}_{c, j}+1\right)
$$




$$
\begin{array}{ll}
\text { subject to } & \mathbf{x}^{c} \leq \mathbf{Z}^{c}, \quad \forall c \in \mathscr{C}, \\
& \mathbf{X}_{j}^{\mathrm{T}} \mathbf{O}_{j} \leq T_{j}, \quad \forall j \in \mathscr{J}, \forall c \in \mathscr{C}, \\
& \mathbf{x}^{c} \mathbf{1}^{\mathrm{T}} \leq\left(N^{c}\right)^{\mathrm{T}}, \quad \forall c \in \mathscr{C}, \\
& \mathbf{x}^{c} \in\{0,1\}, \quad \forall c \in \mathscr{C} .
\end{array}
$$

The constraint in (21b) ensures that a channel can be allocated to the WSOs registered in $c$ th CM only if the channel is available in their respective region; that is, $x_{w, j}^{c} \in$ $\mathbf{x}^{c}=1$ iff $z_{w, j}^{c} \in \mathbf{z}_{w}^{c}=1$. The constraint in (21c) ensures that the WSOs scheduled in a $j$ th channel preserve the system QoS performance, as defined in (14); that is, the total allocated channel occupancy time of coexisting WSOs must preserve the channel window time. The constraint in (21d) ensures that the number of channels allocated to the $c$ th CM is restricted by the number of channels desired by its WSOs. Finally, (21e) forces the decision variable to be binary valued. The constraints in (21e) and (21c) help the system in (1) to satisfy the third objective of TVWS sharing problem in Section 3.2. The optimization problem in (21a)-(21e) seeks to optimize a concave objective function over a convex set. The problem in (21a)-(21e) has a unique solution; as from the optimization theory [39], maximizing a concave function over a convex set has a unique solution. A solution approach to the problem in (21a)-(21e) is presented in the following section.

\section{Solution Method}

The nonlinear objective function (21a) and binary-valued constraint (21b) make the problem in (21a)-(21e) a nonlinear combinatorial optimization problem. Determining the optimal solution of such a problem is a challenging task as the problem becomes intractable as the number of discrete variables increases [40]. Therefore, to ease the solution approach, the problem in (21a)-(21e) is transformed into a linear programming problem with relaxed binary constraint.

5.1. Linearization. The objective function (21a) is linearized using a piecewise linear approximation. In this process, tangent line approximation is used to approximate the objective function in (21a), denoted as $F$. The detailed description of linear approximation is provided in Appendix A. Using this function, the problem in (21a)-(21e) is linearized as

$$
\begin{aligned}
& \max \quad \sum_{c \in \mathscr{C}} \sum_{j \in \mathcal{J}} F\left(\mathcal{U}_{c, j}\right) \\
& \text { subject to } \quad \mathbf{x}^{c} \leq \mathbf{Z}^{c}, \quad \forall c \in \mathscr{C} \\
& \mathbf{x}_{j}^{\mathrm{T}} \mathbf{O}_{j} \leq T_{j}, \quad \forall j \in \mathscr{J}, \forall c \in \mathscr{C} \\
& \mathbf{x}^{c} \mathbf{1}^{\mathrm{T}} \leq\left(N^{c}\right)^{\mathrm{T}}, \quad \forall c \in \mathscr{C} \\
& \mathbf{x}^{c} \in\{0,1\}, \quad \forall c \in \mathscr{C} .
\end{aligned}
$$

To tackle the binary-valued constraint (22b), we apply Lagrangian relaxation as explained in the following.
5.2. Lagrangian Relaxation. Lagrangian relaxation [41] relaxes a subset of constraints by adding them to the objective function with a penalty term called the Lagrangian multiplier. Let $\lambda:=\left[\lambda_{w, j}\right]_{W \times J}$ be the Lagrangian multipliers matrix. Then, the relaxed problem can be defined as

$$
\begin{array}{cl}
\max _{\mathbf{X}} \quad P(\mathbf{X}, \boldsymbol{\lambda}) \\
& =\sum_{c \in \mathscr{C}} \sum_{j \in \mathscr{J}} F\left(\mathscr{U}_{c, j}\right)+\lambda^{\mathrm{T}}\left(\mathbf{Z}^{c}-\mathbf{x}^{c}\right) \\
\text { subject to } \quad \mathbf{X}_{j}{ }^{\mathrm{T}} \mathbf{O}_{j} \leq T_{j}, \quad \forall j \in \mathscr{J}, \forall c \in \mathscr{C} \\
& \mathbf{x}^{c} \mathbf{1}^{\mathrm{T}} \leq\left(N^{c}\right)^{\mathrm{T}}, \quad \forall c \in \mathscr{C} \\
& \mathbf{x}^{c} \in\{0,1\}, \quad \forall c \in \mathscr{C} .
\end{array}
$$

For a given $\lambda$, the Lagrangian relaxation can be defined as

$$
h(\boldsymbol{\lambda})=\max _{\mathbf{X}} \quad\{P(\mathbf{X}, \boldsymbol{\lambda}) \text { : constraints }(23 \mathrm{~b}),(23 \mathrm{c}),(23 \mathrm{~d})\} .
$$

Then the generalized dual problem of the relaxed problem is defined as follows:

$$
L^{*}=\min _{\lambda} \quad\{h(\lambda): \lambda \geq 0\} .
$$

The solution to (24) is the upper bound of the solution to the original problem (23a)-(23d). Note that (24) is a concave function. For a concave function, a gradient-based approach is generally used to compute a value as close as desired to the optimal value. Thus, if $h$ would have been differentiable, we can use a gradient descent method to have a convergence toward the optimal value. The proposed problem, however, cannot be solved using a gradient descent method. It is because the objective function is piecewise linear which is nondifferentiable at the intersection point of adjacent linear pieces, but subdifferentiable at this point. The subdifferential of $h(\boldsymbol{\lambda})$ at such a point is the set of all subgradients at that point. Thus, we need to compute a sequence of $\left\{\lambda^{k}\right\}_{k \in \mathbb{N}}$ such that either $h\left(\lambda^{k}\right)$ converges to the optimal solution using the subgradient method, which is given in the following dual algorithm. The convergence property of the subgradient algorithm is presented in Appendix B.

5.3. Subgradient Algorithm for Lagrangian Relaxation Based TVWS Sharing Problem. The algorithm defined in Algorithm 1 can be described as follows. In Step 0, the input parameters to the algorithm are defined as follows. The initial values of $\lambda^{0}$ are defined randomly. The parameter $\rho$ is used in defining step size $t^{k}$, defined in the range $\rho^{\min }<\rho \leq 2$ [41]. $\rho_{\text {iter }}$ with upper limit of $\rho^{\text {max iter }}$ counts the number of iterations after which the parameter $\rho$ is updated. $k^{\max }$ is defined as stopping criteria for the algorithm.

The algorithm uses variables initialized in Step 0 to apply a linear programming (LP) solver to solve the dual problem and obtain the $k$ th iteration allocation matrix $\mathbf{X}_{k}$. LP solvers are available on both the commercial and freeware basis. The entries in $\mathbf{X}_{k}$ are then adjusted based upon the corresponding entries in $\mathbf{Z}^{c}$ such that $x_{w, j}^{c} \in \mathbf{x}_{k}^{c}, \forall \mathbf{x}_{k}^{c} \in \mathbf{X}_{k}$ are set equal to 
Step 0. (a) Choose initial values of $\lambda^{0}$.

(b) Set parameters, for example, $\rho=2.0, \rho^{\min }=0.001, \rho_{\text {iter }}=0, \rho^{\max \text { iter }}=5, k=0, k^{\max }=10, F^{\text {best }}=0, h^{\text {best }}=-\infty$, $h^{\text {upper }}=0, \mathbf{X}_{k}^{\prime}=[0]_{W \times J}$.

Step 1. (a) Increment as $k=k+1, \rho_{\text {iter }}=\rho_{\text {iter }}+1$

(b) Given $\lambda^{k}$, solve the relaxed problem using any linear programming technique and obtain $\mathbf{X}_{k}$.

Step 2. Validate $\mathbf{X}_{k}$ as: set $x_{w, j}^{c}:=0$ if $z_{w, j}^{c}=0$.

Step 3. Perform frequency reuse as in Algorithm 2 and get $\mathbf{X}_{k}^{\prime}$.

Step 4. Use $\mathbf{X}_{k}^{\prime}$ to compute the value of the function in (23a), called as $F$, and fairness index value $H$ in (29).

If $F>F^{\text {best }}: F^{\text {best }}=F, h^{\text {upper }}=F^{\text {best }}$ and $\mathbf{X}=\mathbf{X}_{k}^{\prime}$.

Step 5. (a) Use $\mathbf{X}_{k}^{\prime}$ to compute:

(i) Subgradient vector as, $\nabla h\left(\lambda^{k}\right)=\left[\partial h / \partial \lambda_{w, j}^{k}, \forall w\right]$,

(ii) Dual objective in (25),

(iii) Step size as, $t_{k}=\rho\left(h^{\text {upper }}-h\left(\lambda^{k}\right)\right) /\left\|\nabla h\left(\lambda^{k}\right)\right\|^{2}$.

(b) Update the dual variable as, $\lambda^{k+1}=\max \left\{\lambda^{k}+t_{k} \nabla h\left(\lambda^{k}\right), 0\right\}$

Step 6. If $h^{\text {best }}<h\left(\lambda^{k}\right)$ then $h^{\text {best }}=h\left(\lambda^{k}\right)$

else if $\rho_{\text {iter }}>\rho^{\max \text { iter }}$ then $\rho=\max \left\{\rho / 2, \rho^{\min }\right\}$ and $\rho_{\text {iter }}=0$.

Step 7. If $t_{k}<0.001$ or $k>k^{\max }$ stop; otherwise, go to Step 1 .

Algorithm 1: Subgradient algorithm for relaxed TVWS sharing problem.

zero if the corresponding element $z_{w, j}^{c} \in \mathbf{z}_{w}^{c}, \forall \mathbf{z}_{w}^{c} \in \mathbf{Z}^{c}$, is zero. This validation ensures the constraint in (21b).

The algorithm then applies the FR process in Step 3 in Algorithm 1. In this process, the algorithm makes use of the current allocation vector, $\mathbf{X}_{k}$, and interference matrix, as shall be discussed in Section 5.4, to identify a set of WSOs which do not get the channel. The algorithm then repeatedly applies LP solver to perform channel allocation to the unallocated WSOs such that they do not cause interference to the allocated WSOs of neighboring CMs. The FR process is detailed in Section 5.5. The outcome of FR process is an updated allocation matrix $\mathbf{X}_{k}^{\prime}$ which is then used to compute the function values in (23a) and the fairness in allocation among neighboring CMs.

Several fairness measures or metrics are used in the literature to determine whether networks are receiving a fair share of spectrum or not, for example, max-min fairness, Jain's fairness index, fairly shared spectrum efficiency, worstcase fairness. In this paper, we adopt Jain's fairness index [42] to measure fairness in allocation among neighboring CMs. The reason is that it satisfies the desired properties of fairness measure like population size independence, continuity, and so forth, as listed in [43]. These properties are important to be considered in measuring the fairness in allocation. For example, the continuity property shows any slight change in the allocation of individual WSO. Thus, an inefficient use of the TVWS is identified by the fairness index as a WSO with bad channel characteristics gets a high proportion of the spectrum. It is ensured through the use of the continuous allocation metric like fraction of throughput demand, as defined in (26). Such an allocation metric is suitable to measure the fairness in allocation for the case where WSOs demand unequal channel bandwidth [43]. Therefore, based on the fraction of throughput demand of CMs, an allocation metric is defined as follows:

$$
T^{c}=\frac{d^{c}}{d^{\prime c}}, \quad \forall c \in \mathscr{C},
$$

where $d^{c}$ and $d^{\prime c}$ represent the maximum data the $c$ th CM desire to transmit and it can transmit using its allocated channels, respectively. These terms are defined as follows. Let the maximum data that the $c$ th $\mathrm{CM}$ can transmit using its allocated channels be defined in terms of the data that the WSOs registered in it can transmit, defined as follows:

$$
d^{c}=\sum_{j \in \mathscr{J}} \sum_{w \in \mathscr{W}^{c}} x_{w, j}^{c} O_{w, j}^{c} r_{w, j}^{c}, \quad \forall c \in \mathscr{C} .
$$

Note that channels are considered as additive white Gaussian noise (AWGN). The data the CM desires to transmit is defined as

$$
d^{\prime c}=\sum_{j \in \mathcal{J}} \sum_{w \in \mathscr{W}} O_{w, j}^{c} r_{w, j}^{c}, \quad \forall c \in \mathscr{C} .
$$

The normalized throughput vector $\left(T^{1}, \ldots, T^{C}\right)$ is then adopted to measure fairness in allocation using Jain's fairness index [42] as

$$
H\left(T^{1}, T^{2}, \ldots, T^{C}\right)=\frac{\left(\sum_{c \in \mathscr{C}} T^{c}\right)^{2}}{C \sum_{c \in \mathscr{C}}\left(T^{c}\right)^{2}} .
$$

Function $H$ in (29) outputs a value in the range of $[0,1]$; when the value is closer to 1 , the allocation is deemed fairer.

If the current iteration value of the objective function, $F$, is optimal, then $F^{\text {best }}$ is updated with $F$ and $\mathbf{X}$ with $\mathbf{X}_{k}^{\prime}$. As the iteration progresses, the feasible primal $F^{\text {best }}$ and lower bound $h^{\text {best }}$ approach gradually the integer optimal by adjusting $\lambda^{k}$ using the subgradient method as defined in Step 5. In Step 5, the subgradient vector of the objective function and the Lagrangian multiplier vector $\lambda^{k}$ for the $k$ th iteration are calculated. The step size $t_{k}$ is used to calculate the multiplier vector for the next iteration. The Lagrange multipliers are thus adjusted iteratively. The convergence property of the subgradient algorithm is discussed under Appendix B. The algorithm terminates as one of the termination conditions is satisfied: 
Input: $\lambda^{k}, \mathbf{X}_{k}^{\prime}=\mathbf{X}_{k}, \mathscr{Z}, \mathrm{CS}$.

Output: $\mathbf{X}_{k}^{\prime}$

Step 0. Given CS generate encoded CS, that is, $\delta_{w, j}, \forall w \in \mathscr{W}, \forall j \in \mathscr{J}$ and interference matrix $\mathbf{Y}$, as defined in Section 5.4.

Step 1. Given $\mathbf{X}_{k}^{\prime}$, update $\mathbf{y}(j) \in \mathbf{Y}, \forall j \in \mathscr{J}$ as: For each $w$ th WSO do: if $x_{w, j}=1: I_{w, m}(j)=0, \forall m \in \mathscr{W}, \forall j \in \mathscr{J}$ or if

$\left(x_{m, j}=1\right.$ and $\left.w \in \mathcal{S}_{m, j}\right): I_{w, m}(j)=0, \forall m \in \mathscr{W}$.

Step 2. Define unallocated WSO set in the system as, $\mathscr{W}^{\prime}=\left\{\forall w \in \mathscr{W}: \exists j \in \mathscr{J} \mid \sum_{m \in \mathscr{W}} I_{w, m}(j)>0\right\}$.

Step 3. While $\sum_{w \in \mathscr{W}} \sum_{m \in \mathscr{W}} I_{w, m}(j)>0, \forall j \in \mathscr{J}$ and $\mathscr{W}^{\prime} \neq\{\}$ do

(a) Given $\lambda^{k}$, and $\mathscr{W}^{\prime}$; solve the relaxed problem using any linear programming solver and obtain $\mathbf{X}_{k}$.

(b) Perform following updates:

(1) Update $\mathbf{X}_{k}$ as, $x_{w, j}^{c}:=0$ if $z_{w, j}^{c}=0$.

(2) Update $\mathbf{X}_{k}^{\prime}$ as, $\mathbf{X}_{k}^{\prime}=\mathbf{X}_{k}^{\prime}+\mathbf{X}_{k}$.

(3) Update $\mathscr{W}^{\prime}$ as, $\mathscr{W}^{\prime} \leftarrow \mathscr{W}^{\prime} \backslash\left\{\forall w \in \mathscr{W}^{\prime} \mid \exists j \in \mathscr{J}: x_{w, j}^{c}=1\right\}$.

(4) Update $\mathbf{Y}$ as in Step 1.

Algorithm 2: Subroutine: frequency reuse method.

(i) Dual step size becomes less than a set threshold or

(ii) the number of iterations exceeds the maximum number of iterations.

After the overall iteration ends, we regard the final value of $F^{\text {best }}$ as the approximated optimal solution and the corresponding allocation matrix $\mathbf{X}$ is the algorithm output.

The interference matrix, $\mathbf{Y}$, that is used to implement the FR step in Algorithm 2 is defined in the following section.

5.4. Interference Matrix. The WSOs registered in the neighboring CMs and interfering on the available TV channels are represented using an interfering matrix called $Y$-matrix in this paper. Note that the $Y$-matrix does not model the interference among coexisting WSOs. Rather, it represents the set of WSOs which cannot transmit simultaneously on the available TVWS due to interfering transmission regions. In fact, in IEEE 802.19.1 [10], a coexistence discovery algorithm is presented that the CDIS and CM run to perform the statistical analysis of the expected interference among coexisting WSOs. Briefly, the algorithm in [10] takes the WSOs' geographic location, transmitter and receiver characteristics, antenna height and directivity, height above average terrain, and other related parameters to execute interference discovery process. In this process, a cumulative distribution function of the potential interference from $m$ th WSO to $w$ th WSO is estimated. Both of these, $m$ th and $w$ th WSOs, could register to the same $\mathrm{CM}$ or different $\mathrm{CMs}$ in the system. The minimum interference level, experienced by $90 \%$ devices of the $w$ th WSO, is then taken as the potential interference value from an $m$ th WSO to $w$ th WSO. The measured interference value is then compared to a threshold. If the value is greater than the threshold, the $m$ th WSO is considered potential interferer to the $w$ th WSO and is included in its CS. A similar rule is applied for interference discovery of the $w$ th WSO into the $m$ th WSO. Thus, the outcome of the interference analysis process is a CS of each WSO registered in the CMs in the system. The system in (1) then makes use of the CS of each WSO to generate a $Y$-matrix as follows.

Let a set $\mathcal{S}_{w, j}=\left\{I_{w, m}(j)\right\}, \forall m \in \mathscr{W}$, be an encoded CS of $w$ th WSO on $a$ th channel such that an indicator variable
$I_{w, m}(j)=1$ if $m$ th WSO interferes $w$ th WSO transmission on the $j$ th channel, as defined in Table 1; otherwise $I_{w, m}(j)=0$. The encoded CS of all the WSOs coexisting on $j$ th channel are then used to define a $j$ th channel interference matrix $\mathbf{y}(j)$ as follows:

$$
\begin{aligned}
& \mathbf{y}(j) \\
& :=\left[\begin{array}{cccccc}
\times & I_{1,2}(j) & \cdots & I_{1, w}(j) & \cdots & I_{1, W}(j) \\
& \vdots & & \vdots & \\
I_{w, 1}(j) & I_{w, 2}(j) & \cdots & \times & \cdots & I_{w, W}(j) \\
& & \vdots & & \vdots & \\
I_{W, 1}(j) & I_{W, 2}(j) & \cdots & I_{W, w}(j) & \cdots & \times
\end{array}\right],
\end{aligned}
$$

where $x$ in diagonal vector in $\mathbf{y}(j)$ represents don't care condition. This condition translate a self-interference indicator variable, $I_{w, w}(j)$, having no meaning. The $w$ th row in $\mathbf{y}(j)$ matrix represents encoded CS of $w$ th WSO. The interference matrices for all channels in the system are then used to define an interference matrix $\mathbf{Y}$ as follows:

$$
\mathbf{Y}=\left[\begin{array}{llll}
\mathbf{y}(1) & \mathbf{y}(2) & \cdots & \mathbf{y}(J)
\end{array}\right]
$$

The TVWS sharing algorithm in Algorithm 1 makes use of the interference matrix $\mathbf{Y}$ to implement FR in sharing TVWS among heterogeneous WSOs, as discussed in the following subsection.

5.5. Frequency Reuse. The frequency reuse (FR) subroutine in Algorithm 2 performs spatial reuse of the TV spectrum to enhance its effective utilization. The FR process is implemented to the WSOs that do not get channel in the initial allocation phase in Step 1, Algorithm 1. This requires identifying a set of unallocated WSOs eligible for the FR. In this process, an encoded CS $\mathcal{S}_{w, j}, \forall m \in \mathscr{W}$, and an interference matrix $\mathbf{Y}$ are used to define the set of unallocated WSOs, $\mathscr{W}^{\prime}$. To generate encoded CS and $Y$-matrix, we make use of the CS of each WSO available at MCM. Note that the 802.19.1 defines different message clauses that enable 
CMs to exchange their WSO related information [10]. Let us assume that the CS of WSOs are available to CDM at MCM. Given such information available, an encoded CS of WSOs, $\mathcal{S}_{w, j}, \forall m \in \mathscr{W}$, and an interference matrix, $\mathbf{Y}$, are generated, as defined in Section 5.4. Initially the $Y$-matrix is filled with all ones. Let $\mathbf{X}_{k}$ be an initial allocation matrix available from Step 2, Algorithm 1. The $Y$-matrix is then updated based on $\mathbf{X}_{k}$ and $\mathcal{S}_{w, j}, \forall m \in \mathscr{W}$, in Step 1, Algorithm 2, as follows. For each $j$ th channel in the system, update interference matrix $\mathbf{y}(j) \in \mathbf{Y}$ as follows.

(1) If $j$ th channel is allocated to $w$ th WSO, set all $w$ th row elements in $\mathbf{y}, \forall \mathbf{y} \in \mathbf{Y}$ equal to zero or

(2) if $j$ th channel is allocated to $m$ th WSO and $w$ th WSO is in the CS of $m$ th WSO, set all $w$ th row elements in the matrix $y$ equal to zero.

The above two steps identify the eligibility of the WSOs for implementing the FR process. For example, if the $w$ th WSO is already allocated a channel, we aim to restrict it in taking part the FR process. Therefore, the $w$ th row entries in the entire $Y$-matrix are flipped zero in the first step above. Similarly, if a $j$ th channel is already allocated to $m$ th WSO and if $w$ th WSO transmission in the $j$ th channel shall create harmful interference to the $m$ th WSO transmission, the $j$ th channel cannot be spatially reused at unallocated $w$ th WSO. Therefore, $Y$-matrix entries corresponding to $w$ th row are also flipped zero. The updated $Y$-matrix thus defines a set of unallocated WSOs. These are the WSOs for which at least one nonzero entry exists in the corresponding row in the $Y$ matrix, as defined, in Step 2, Algorithm 2.

The subroutine in Step 3, Algorithm 2, then repeatedly allocates the available TV channels to the WSOs in the set $\mathscr{W}^{\prime}$ as follows. The relaxed problem in (24) is solved using any LP solver for the WSOs in the set $\mathscr{W}^{\prime}$ and an allocation matrix $\mathbf{X}_{k}$ is obtained. $\mathbf{X}_{k}$ is then used to update $\mathbf{X}_{k}^{\prime}, \mathscr{W}^{\prime}$, and $Y$-matrix, as defined in Steps 3(b)(2), 3(b)(3), and 3(b)(4), respectively. This repetitive update and allocation process continues until all WSOs in the set $\mathscr{W}^{\prime}$ get the channel or no more FR is possible.

Let us apply the FR implementation in the coexisting scenario shown in Figure 3. In this figure, four WSOs operating in three network technologies, an IEEE 802.22 regional area network, IEEE 802.11 local area networks, and IEEE 802.15.4 personal area network, are deployed in some geographic region. The shaded area around each transmitter denotes its transmission radius. The circular links between a transmitter and receivers show wireless connectivity between them. The receiver nodes in some networks receive interfering signals from other collocated transmitters as shown in the figure. Let WRAN, HS1, HS2, and PAN be labelled as, WSO 1, 2, 3, and 4, respectively. Let us assume each of the WSOs is registered in a dedicated CM; that is, four neighboring CMs are available in the CDM system. Let us suppose that a single TV channel is available in the region for secondary use. Then, based on coexisting scenario shown in the figure, the encoded CS of each WSO can be defined as follows:

$$
\begin{aligned}
& \mathcal{S}_{1,1}=\{0,1,0,0\}, \\
& \mathcal{S}_{2,1}=\{1,0,1,1\},
\end{aligned}
$$

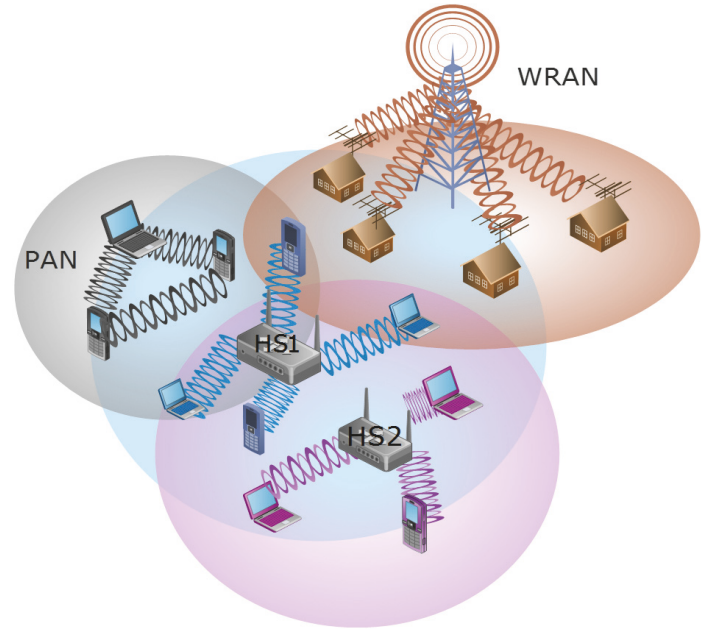

FIGURE 3: IEEE 802.22 wireless regional area network (WRAN), IEEE 802.11 hotspots (HS1, HS2), and IEEE 802.15.4 personal area network (PAN) coexisting in some geographic region.

$$
\begin{aligned}
& \mathcal{S}_{3,1}=\{0,1,0,0\}, \\
& \mathcal{S}_{4,1}=\{0,1,0,0\} .
\end{aligned}
$$

The $Y$-matrix is then populated from the bitwise OR operation on the CS of the WSOs. The generated $Y$-matrix is $\mathbf{Y}=\left[\begin{array}{llll}1 & 1 & 1 & 1\end{array}\right]$. Let for some given input parameters, as listed in Table 1, the algorithm in Algorithm 1 find an initial allocation vector, $\mathbf{X}=\left[\begin{array}{llll}1 & 0 & 1 & 0\end{array}\right]$. The allocation vector shows that WSO 1 and WSO 3 are allocated the channel. The FR process is then invoked. The $Y$-matrix is updated to identify WSOs eligible for spatially reusing the channel, as follows. The XOR operation is performed as $(\mathbf{Y}=\mathbf{X} \oplus \mathbf{Y})$. This operation turns the entries in $Y$-matrix equal to zero where the corresponding entries in $X$-matrix are ones. The $Y$-matrix at this stage looks like $\mathbf{Y}=\left[\begin{array}{llll}0 & 1 & 0 & 1\end{array}\right]$. It is then updated using the CS of allotted WSOs as previously defined in the second rule of $Y$-matrix update. The second entry in $Y$-matrix is thus flipped zero as WSO 2 is in the CS of allotted WSO 1. The updated $Y$-matrix then looks like $\mathbf{Y}=\left[\begin{array}{llll}0 & 0 & 0 & 1\end{array}\right]$. The algorithm then solves the dual problem again and allocates the channel to WSO 4. The final allocation matrix then looks like $\mathbf{X}=\left[\begin{array}{llll}1 & 0 & 1 & 1\end{array}\right]$. The final allocation shows that the available TV channel is reused at WSO 4 without causing harmful interference to allotted WSO 1 and WSO 3.

5.6. Scheduling Map. Once the allocation process in Algorithm 1 and frequency reuse in Algorithm 2 terminates, the CDM system generates a scheduling map to send it to the CMs in the system. The scheduling map (SM) is a map showing the WSOs' scheduling periods arranged in window time in the allocated channels. In this paper, the scheduling period of a wth WSO refers to its channel timeslot, that is, COT. For example, SM of three WSOs scheduled in an allocated TV channel is shown in terms of their COT defined in the window time in Figure 2. Thus, given the COT of 
WSOs and the allocation matrix $\mathbf{X}$, from the algorithm in Algorithm 1, the SM is a simple procedure of defining two timing parameters: transmission start time and transmission end time. The CDM system defines the timing parameters for WSOs registered in the CMs in the system as follows.

Let a pair of transmission variables $\left(t_{w, j}^{\text {start }}, t_{w, j}^{\text {stop }}\right)$ precisely define the time instance in which the $w$ th WSO, registered in $c$ th CM, may start and stop its transmission on an allotted $j$ th channel, respectively. $t_{w, j}^{\text {start }}$ and $t_{w, j}^{\text {stop }}$ are calculated as follows. Let a variable $C_{w, m(w)}$ be defined as the cost of sharing a channel between two WSOs, $w, m \in \mathscr{W}$, where $m(w)$ represents a WSO $m$ sharing a channel with WSO $w$. Let $\tau_{w}$ represent the control overhead associated with MAC technology of the $w$ th WSO. The control overhead is defined as the amount of time required to perform control signaling while operating in the TVWS. This value is fixed and predetermined based upon the underlying network technology of the WSO. For example, if an 802.22 WSO employs OFDMA, one OFDM symbol is used for both the frame preamble and the frame header, except for the first frame in the superframe which consumes two additional symbols ( $1 / 4$ cyclic prefix mode). If we consider two OFDM symbols per frame as a control region then using a symbol duration, $T_{\text {Sym }}=0.3733 \mathrm{~ms}$ [44], the control overhead per frame is computed as $0.7466 \mathrm{~ms}$. Other settings may generate different overhead. Similarly, if a WSO $m$ operates in a different network technology than that of the WSO $w$, its control overhead will be different from that of WSO $w$. The total overhead in a channel varies as the channel is shared among heterogeneous WSOs. The value of the parameter $C_{w, m(w)}$ is then defined simply by adding the control overhead of all WSOs sharing a channel as follows:

$$
\begin{aligned}
& C_{w, m(w)} \\
& := \begin{cases}\tau_{w}+\tau_{m} & \text { if } \mathrm{MAC}_{w} \neq \mathrm{MAC}_{m}, \forall(w, m) \in \mathscr{W}^{\prime c} \\
0 & \text { otherwise }\end{cases}
\end{aligned}
$$

where $\mathscr{W}^{\prime c} \subset \mathscr{W}^{c}$ refers to the set of WSOs with NID listed before NID of $w$ th WSO in $\mathscr{W}^{c}$. The timing parameters are computed as

$$
\begin{aligned}
t_{w}^{\text {start }} & =\sum_{m \in \mathscr{W}^{\prime c}} O_{m, j}^{c} x_{m, j}^{c}+C_{m, m(w)}, \\
t_{w}^{\text {stop }} & =t_{w}^{\text {start }}+O_{w, j}^{c} .
\end{aligned}
$$

Thus, $t_{w, j}^{\text {start }}$ refers to the time instance in the scheduling window that all the WSOs $m$ have utilized the channel for the duration of their respective COT. Note that in defining the scheduling map we make a simplifying assumption that the timers of WSOs in the system are presynchronized and WSOs sharing a $j$ th channel have agreed on the reference time (the time instance in which the window time starts) as defined in [10]. Timer synchronization may be done by having agreements between service providers managing the WSOs which is outside the scope of this paper.

The CDM defines SM and sends it to the SCMs. The SCMs send the SM to the registered WSOs. Such implementation shall reduce the control signaling between the WSOs and the pertinent CM. The control signaling is otherwise inevitable while performing context switching among WSOs scheduled in the TV channel. Once the spectrum has been allocated, the SM remains unchanged unless (i) an incumbent appears in one of the assigned channels and (ii) a change in a WSO's channel occupancy demand or some other coexisting WSO's demand requires readjusting the WSO's allocation.

\section{Simulations and Analysis}

The performance of the proposed channel sharing algorithm is compared with two other channel allocation algorithms, proposed in $[17,18]$.

6.1. Comparative Channel Allocation Schemes. In this section, we summarize the allocation mechanism of the comparative TVWS allocation schemes. In [17], two TVWS sharing problems are defined: one for maximizing the number of channels allocated to the networks and the second for maximizing the total throughput under the minimum fairness constraint of allocating at least a single channel to each network. In this simulation setup, we implement the second problem as it closely matches with the channel sharing scheme proposed in this paper. The TVWS sharing algorithm proposed in [17] then selects a node (WSO) having a minimum of the assigned channels and the minimum number of the available channels to it. The algorithm assigns a TV channel to the selected WSO and calculates the total throughput. It keeps assigning the channel to other WSOs as long as the total throughput is increasing. This procedure is repeated for every channel. The algorithm terminates as no more increase in the throughput is observed.

The TVWS sharing problem in [18] is modeled as a lexicographic ordering of throughputs of access points of coexisting networks. The proposed problem is then transformed into a graph coloring problem. An algorithm called Share is then proposed to solve the graph coloring problem. The Share algorithm operates in three phases. In the first phase of allocation, it orthogonalizes the WSOs in the available TV channels (frequency slots). In the second phase, a mutual channel sharing is performed among allotted WSOs of the first phase under the condition that their first phase throughputs do not decrease. The fairness is improved in the third phase by sharing the channel with unallocated WSOs such that lexicographically ordered throughputs do not decrease.

We select the algorithms in $[17,18]$ due to the close resemblance of their TVWS sharing problems to the proposed channel sharing mechanism. For example, both consider optimizing throughput under minimum fairness in allocation. However, there exist some fundamental differences as well. For example, both the allocation schemes orthogonalize the WSOs in frequency domain by allocating a dedicated channel to each allocated WSO, while the proposed scheme orthogonalizes WSOs in a joint time-frequency domain by slicing the available TVWS in the frequency bands and further slicing each channel (frequency band) into a number of COTs in the channel window time, as discussed in Section 4. Moreover, the algorithm in [17] is intended for 
TVWS channel allocation to cellular networks, while the proposed scheme is intended for TVWS sharing in an ad hoc coexisting environment, as discussed in Section 1. Similarly, the TVWS sharing algorithm in [18] does not implement the FR concept. Therefore, we implement the proposed algorithm without FR process as well to have a fair comparison with the scheme in [18]. This is achieved by omitting Step 3 in Algorithm 1 during the implementation of the proposed algorithm.

Finally, the performance of the proposed allocation scheme with and without FR implementation is judged in comparison with the scheme in [17] and the scheme in [18], respectively.

6.2. Simulation Setup. Simulation setup consists of 32 WSOs deployed in some geographic region and connected to an 802.19.1 coexistence system. The system has $32 \mathrm{CMs}$, each serving a single WSO. We select a dedicated CM for each WSO as the schemes in $[17,18]$ perform TVWS sharing at network (WSO) level. The number of available TV channels in the region varies from 2 to 16 . The WSO types and transmission powers are modeled using FCC regulations [2]. For this purpose, the specifications for fixed mode 1 and mode 2 WSO types are used. The fixed mode 1 and mode 2 type WSOs are allowed to have maximum antenna gain of 4 watts (W) effective isotropic radiated power (EIRP), $100 \mathrm{~mW}$ EIRP, and $100 \mathrm{mWatt}$ EIRP, respectively. The WSO access technologies are IEEE 802.22 and IEEE 802.11af. In this simulation setup, we implement the compulsory channel requirement of each WSO where the standard definition of the above technologies mandates a single TV channel of regulatory defined bandwidth as a requirement of a device to operate in the TVWS. Note that the bandwidth of a TV channel is set equal to $6 \mathrm{MHz}$.

Two parameters, WSO channel occupancy demand, $O_{w, j}^{c}$, and WSO density in the region, $K_{w, j}^{c}$, are varied to observe their effect on allocation behavior of the three allocation schemes as follows. Let $T_{j}$ represent the window time on the $j$ th channel. Note that the 802.19.1 [10] does not define MAC layer frame structure for operations in TVWS. Therefore, the channel window time is not defined in an absolute time domain in 802.19.1. In this simulation setup, we define the channel window time as a unit length, without loss of generality; that is, $T_{j}=1, \forall j \in \mathscr{J}$. Then, three allocation subdomains are defined on $T_{j}$ as follows; low subdomain consists of up to 33 percent of the channel window time, defined as $O^{L}:=(0,0.33] T_{j}$, a medium subdomain consists of 34 to 67 percent of the channel window time, defined as $O^{M}:=[0.34,0.67] T_{j}$, and a high subdomain consists of 67 to 100 percent of the channel window time, defined as $O^{H}:=$ $[0.67,1] T_{j}$. The channel occupancy demand of each WSO is then randomly defined on these subdomains.

The WSO density in the region is reflected using the number of WSOs in the CS of each WSO as follows. Let $W$ be the number of WSOs registered in all CMs in the system; then we define three WSO density subdomains as low $K^{L}:=(0,0.33] W$, medium $K^{M}:=[0.34,0.67] W$, and high $K^{H}:=[0.67,1] W$. The CS of each WSO is randomly defined on these subdomains. Let $K_{w, j}^{c}$ represent the number of WSOs in the CS of $w$ th WSO on the jth channel, registered in $c$ th CM. Then, the effect of the variability in the translated channel occupancy demand and WSO density is measured using a pair of parameters $\left(O_{w, j}^{c}, K_{w, j}^{c}\right)$. Note that varying each of these parameters on three respective subdomains results in $2^{3}=27$ possible allocation combinations. Out of 27 , we select three cases to study the performance metrics defined in Section 6.3 as follows:

(i) Low: low COT, low WSO density, that is, $O_{w, j}^{c} \in O^{L}$ and $K_{w, j}^{c} \in K^{L}$

(ii) Medium: medium COT, medium WSO density, that is, $O_{w, j}^{c} \in O^{M}$ and $K_{w, j}^{c} \in K^{M}$

(iii) High: high COT, high WSO density, that is, $O_{w, j}^{c} \in O^{H}$ and $K_{w, j}^{c} \in K^{H}$

Next, we apply the intlinprog routine of MATLAB ${ }^{\circledR}$ to solve the proposed TVWS sharing problem. The routine applies the mixed-integer linear programming technique. Since we need binary-valued vector $\mathbf{X}$, therefore, we set all the decision variables, $x_{w, j}^{c} \in \mathbf{X}^{c}, \forall \mathbf{X}^{c} \in \mathbf{X}$, to be integer variables in the intlinprog routine. The binary decision may lead to the situation where the COT of allocated WSOs may not fit the channel window time. For example, let us assume that the WSOs 1, 2, 3, and 4 in Figure 3 coexist in a $\mathrm{TV}$ channel. Let their COT demand be defined as $0.25,0.33$, 0.37 , and 0.15 , respectively. Let us assume the intlinprog routine outcome as $\mathbf{X}=\left[\begin{array}{llll}1 & 0 & 1 & 1\end{array}\right]$; that is, the WSOs 1, 3, and 4 get the channel. This results in total COT of allocated WSOs equal to 0.77 which is less than the channel window time, 1. The second WSO cannot be accommodated in the channel considering the constraint (23b). In this simulation, the solution $\mathbf{X}$ is engineered such that the second WSO is partially allocated to the desired COT so as to maximize the channel utilization while maintaining constraint (23b). In order to have a fair comparison, the same engineering principle is applied to the allocation matrix generated by the comparative allocation schemes. The comparative analysis of the three allocation schemes is then performed as discussed in the following section.

6.3. Comparative Analysis. The relative performance of the three allocation schemes is evaluated using the following metrics: system throughput, fairness in allocation among CMs, and WSO satisfaction from the allocation. These performance metrics are selected to analyze how well the three allocation schemes achieve the TVWS sharing objectives, as defined in Section 3.2. The simulation results of the performance metrics are presented in Figures 4-6, respectively. Subplots (a), (b), and (c) in these figures show the effect of varying $\left(O_{w, j}^{c}, K_{w, j}^{c}\right)$ pair in low, medium, and high subdomains, respectively. The study results are discussed as follows.

6.3.1. System Throughput. Figure 4 shows the system throughput (ST) achieved by the three allocation schemes. 


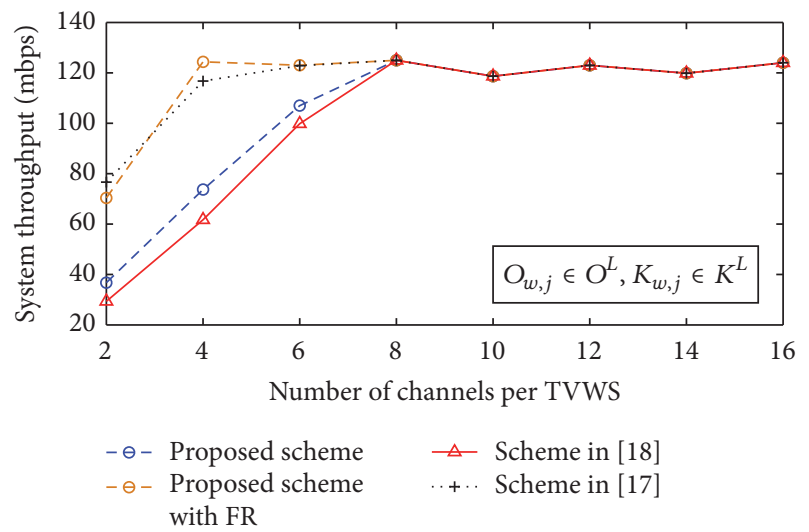

(a)

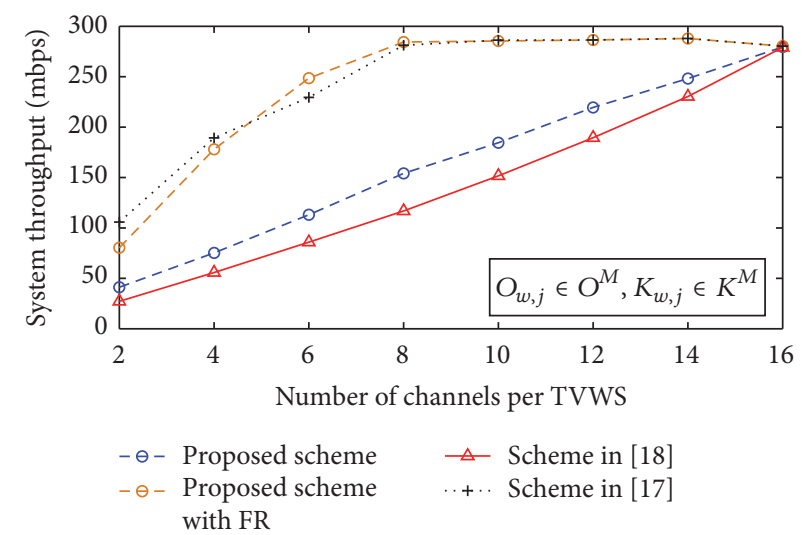

(b)

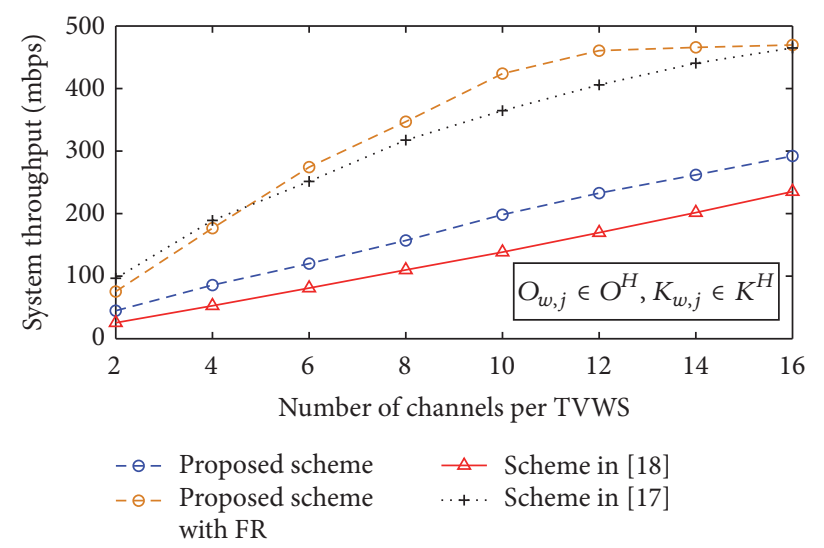

(c)

FIGURE 4: System throughput for 32 WSOs registered in all CMs for a varying number of TV channels in the system.

Given the allocation matrix $\mathbf{X}$ and the SINR values, the ST is defined using Shannon capacity formula [45] as

$$
\mathrm{ST}=\sum_{c \in \mathscr{C}} \sum_{j \in \mathscr{J}} \sum_{w \in \mathscr{W}^{c}} x_{w, j}^{c} O_{w, j}^{c} b_{j} \log _{2}\left(1+\operatorname{SINR}_{w, j}^{c}\right) .
$$

It is shown in Figure 4 that, for most of the channels in the system, the proposed scheme achieves higher ST gain than the comparative TVWS sharing schemes. However, the proposed scheme with FR implementation achieves slightly lower ST than the scheme in [17] for the case when the number of channels in the system is two. This is because the scheme in [17] focuses on maximizing the throughput in the TVWS allocation process, while the proposed scheme focuses on making a balance among the contradicting QoS metrics, ST and fairness in allocation. Consequently, the WSOs with lower channel quality (here lower SINR value) also get a proportion of the available TVWS which reduces the total ST gain in the proposed scheme. However, as the number of channels in the system reaches to four and above, the proposed scheme achieves higher ST gain and remains so until both the schemes converge to the maximum achievable ST. The reason for such improvement is that the proposed scheme applies a joint time-frequency FR concept which accommodates a higher number of WSOs in the available TV channels, while the scheme in [17] applies FR concept in frequency domain only. Note that the ST gain in this study is defined as maximum if all of the WSOs in all the CMs get their desired channel demands.

The effect of variability in the $\left(O_{w, j}^{c}, K_{w, j}^{c}\right)$ pair values on the ST gain of the three allocation schemes is shown in Figures 4(a), 4(b), and 4(c), respectively. The three allocation schemes converge to the maximum ST, as the number of channels in the system reaches 8 and 16, as shown in Figures 4(a) and 4(b), respectively. However, in high subdomain case (Figure 4(c)), none of the allocation scheme achieves the maximum ST. The reason is that the high channel occupancy demand of WSOs results in a few WSOs to saturate the available TVWS while leaving no channel share for rest of the WSOs.

Another notable property of the ST study is that, as the $\left(O_{w, j}^{c}, K_{w, j}^{c}\right)$ pair values increase from low to high subdomains, the ST gain of the proposed scheme improves over ST gains in the comparative scheme, as shown in Figures $4(\mathrm{a})-4(\mathrm{c})$, respectively. This improvement is attributed to the combined effect of the use of the proportional fairness in the allocation and implementing of FR in a joint time-frequency domain in the proposed scheme, as defined in Sections 4 and 5 , respectively.

6.3.2. Fairness. The fairness in allocation among CMs in the system is measured using (29) where the variability in CMs' normalized throughput vector, $\mathbf{T}=\left(T^{1}, T^{2}, \ldots, T^{C}\right)^{\prime}$, 


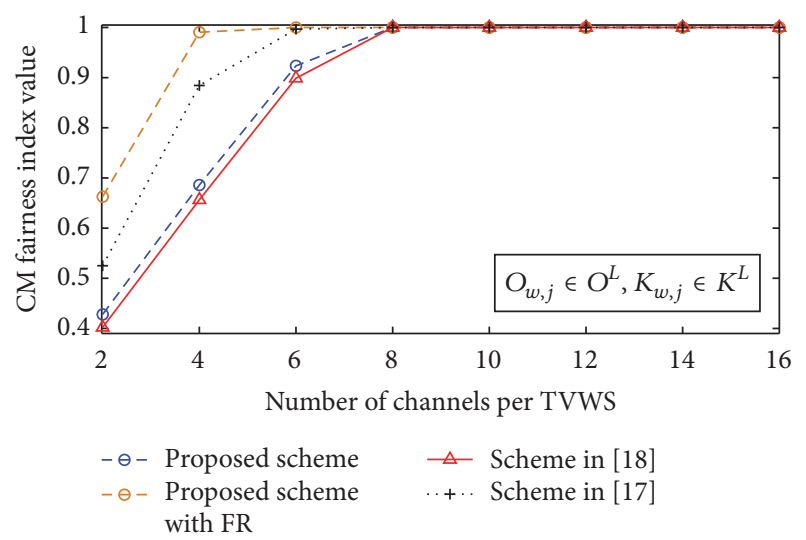

(a)

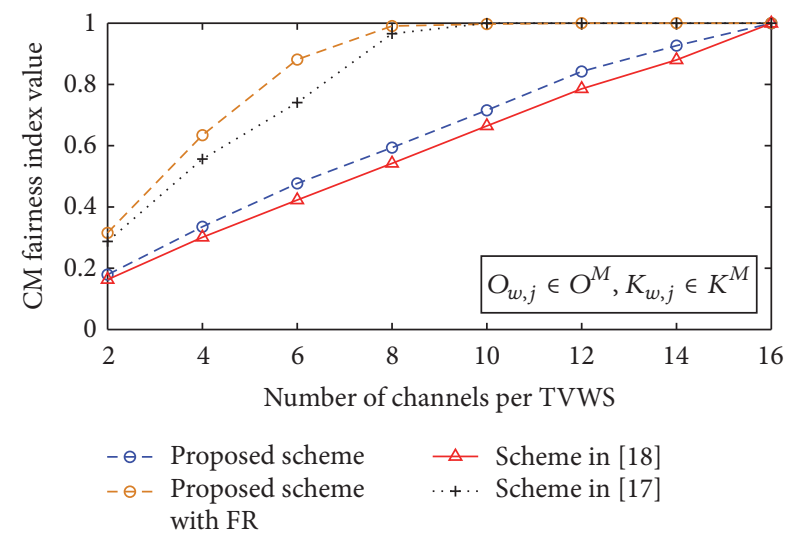

(b)

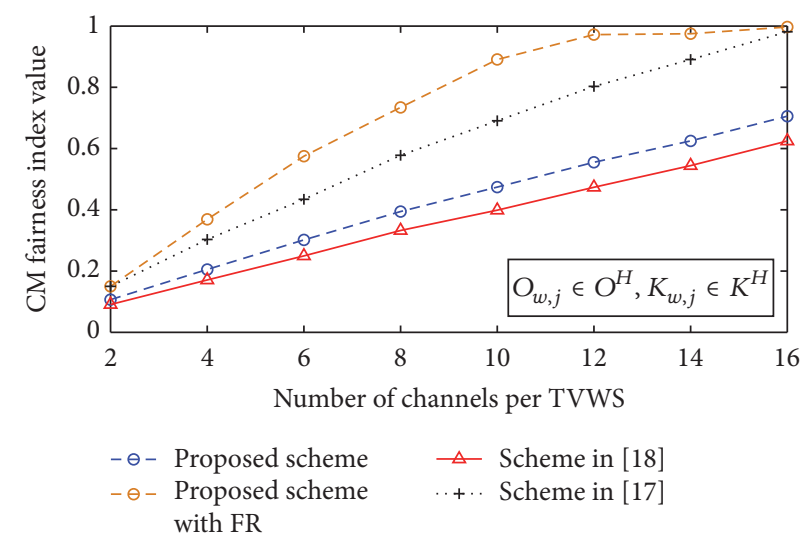

(c)

FIGURE 5: Fairness index value calculated using normalized throughput vector of CMs for a varying number of TV channels in the system.

is used as a fairness metric to compute the fairness index (FI) value. The FI result, as shown in Figure 5, confirms that the proposed scheme achieves the highest FI value due to the combined use of the proportional fairness method and the FR implementation in the joint time-frequency domain. On the other hand, though both the scheme in [17] and the scheme in [18], optimize the fairness in allocation. However, both the schemes make an orthogonal TV channel allocation, thus resulting in lesser number of WSOs to get the channel which reduces FI value. Moreover, the constraint of maintaining the lexicographically ordered throughputs of the WSOs in the scheme in [18] further reduces the degree of the fairness in allocation.

The effect of varying the values of the $\left(O_{w, j}^{c}, K_{w, j}^{c}\right)$ pair in low, medium, and high subdomains is shown in Figures 5(a), 5(b), and 5(c), respectively. It is shown in Figures 5(a) and 5(b) that the FI values of all the comparative allocation schemes converge to the maximum FI value, that is, 1 , as the number of channels in the system reaches 8 and 16, respectively. However, in the high subdomain case (Figure 5(c)), none of the comparative allocation schemes converge to the maximum FI value except for the proposed scheme with the FR implementation. It is because, in all other schemes, their orthogonal channel allocation policy results in a few WSOs to saturate the available TVWS, while in the proposed scheme, the spatial reuse of the TVWS in a joint time-frequency domain accommodates as many as WSOs, registered in the CMs which improves fairness in allocation.

6.3.3. WSO Satisfaction. In this study, we analyze the performance of the three allocation schemes, the third objective of the TVWS sharing problem defined in Section 3.2. In this study, a WSO is considered satisfied from allocation if it gets its desired channel demand for the duration of desired channel occupancy. The system-wide WSO satisfaction percentage $(S)$ is then calculated using percentage of the mean satisfaction as

$$
S=100 \sum_{c \in \mathscr{C}} \frac{\sum_{w \in \mathscr{W}^{c}}\left(\left(\sum_{j \in \mathcal{F}} x_{w, j}^{c}\right) / n_{w}\right)}{\mathscr{W}^{c}}
$$

Figure 6 shows the simulation result of the satisfaction study of the three allocation schemes. This figure shows that the proposed scheme and the scheme in [18] achieve similar satisfaction result as their lines overlap with each other. However, the proposed scheme with FR implementation achieves better satisfaction result than that of the scheme in [17]. It is because the TVWS allocation in a joint timefrequency domain enables the proposed scheme to accommodate as many as WSOs in the available TVWS, while the third objective in the TVWS sharing problem, in Section 3.2, requires the proposed scheme to satisfy the channel demand 


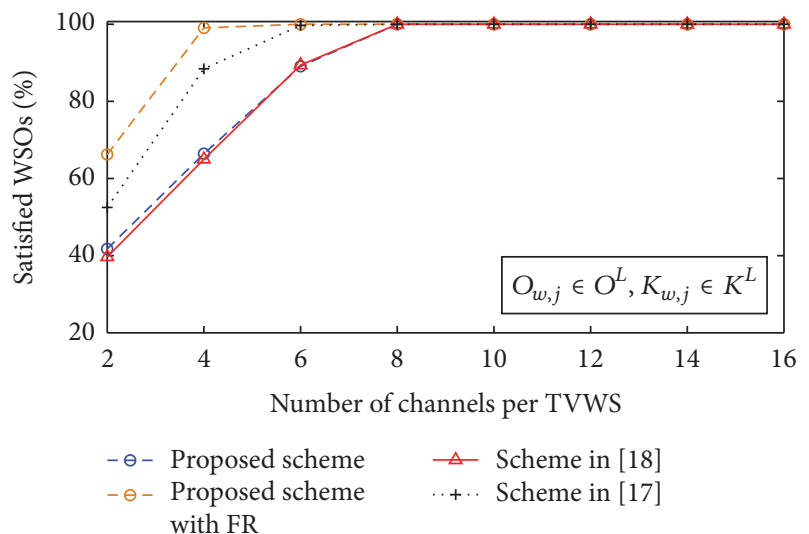

(a)

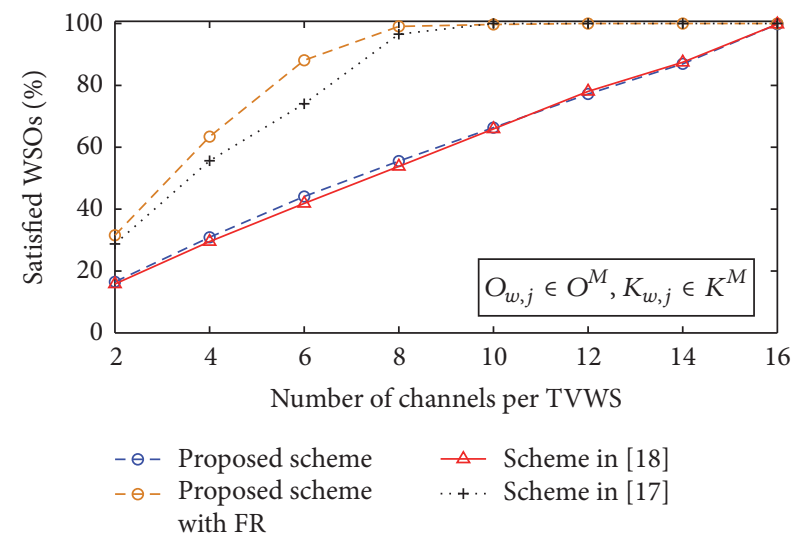

(b)

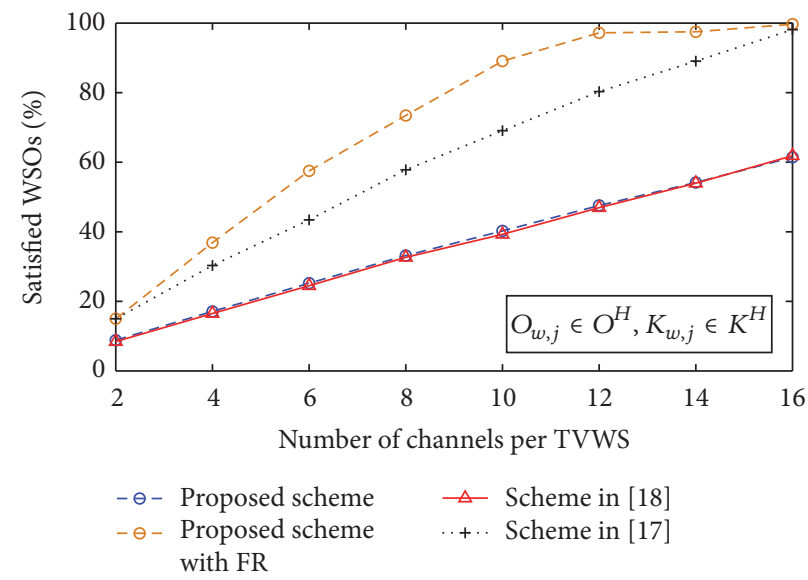

(c)

FIgURE 6: Percentage of total 32 WSOs satisfied from the allocation.

of each allotted WSO. Such an allocation strategy improves the satisfaction result of the proposed scheme.

From the results in Figures 4-6, it is clear that none of the comparative schemes results in better performance than the proposed scheme in any of the performance metrics. The proposed scheme, however, gives fairer channel allocation among all comparative allocation schemes. The proposed scheme with the FR implementation, however, outperforms the comparative schemes, in most of the TV channels in the system, in all the three performance metrics as shown in Figures 4-6.

6.4. Increasing WSO Density. In this section, the effect of increasing the number of coexisting WSOs in the performance of the proposed allocation scheme is evaluated. The performance is measured using the metric like system throughput and WSO satisfaction, for the three subdomain cases, that is, low, medium, and high. The number of WSOs registered in each CM in the system varies in a set, $W \in$ $\{8,16,24, \ldots, 64\}$. The number of available TV channels remains constant at 4 , and the other simulation parameters are the same as defined in Section 6.2. The results of the performance study are shown in Figures 7 and 8.
Figure 7 shows that the highest throughput gain is achieved in the high subdomain case, that is, when $\left(O_{w, j}^{c} \in\right.$ $\left.O^{M}, K_{w, j}^{c} \in K^{M}\right)$. The reason is that the proportional fairness method in the proposed scheme selects the WSOs with high throughput gain to share the available TVWS. Spatially reusing the frequency further helps the proposed scheme to accommodate as many as WSOs in the available TVWS. Consequently, the ST increases in high subdomain case. On the other hand, the achieved throughput is the least in low subdomain case, that is, when $\left(O_{w, j}^{c} \in O^{L}, K_{w, j}^{c} \in K^{L}\right)$. It is because, the low channel occupancy demand of the WSOs could not saturate the available whitespace.

Figure 8 shows the percentage of the number of WSOs satisfied from the allocation, calculated using (36). This figure shows that the satisfaction is the highest in the low subdomain, followed by the medium subdomain, especially in the case when $W=8$, for each CM. The reason is that a relatively greater number of WSOs can be satisfied per TVWS when $W=8$. The WSP value then sharply declines as the number of WSOs in the system increases, especially for the medium and high subdomain cases.

The results in Figures 7 and 8 shall facilitate the modeling of a channel sharing system such that, given the statistics of 


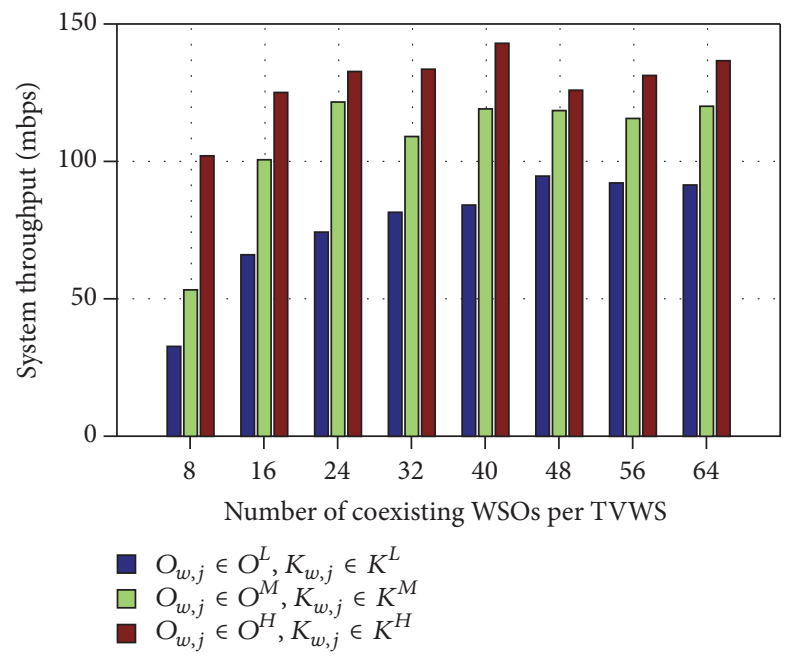

FIGURE 7: System throughput achieved by the proposed scheme for $4 \mathrm{TV}$ channels and a varying number of WSOs.

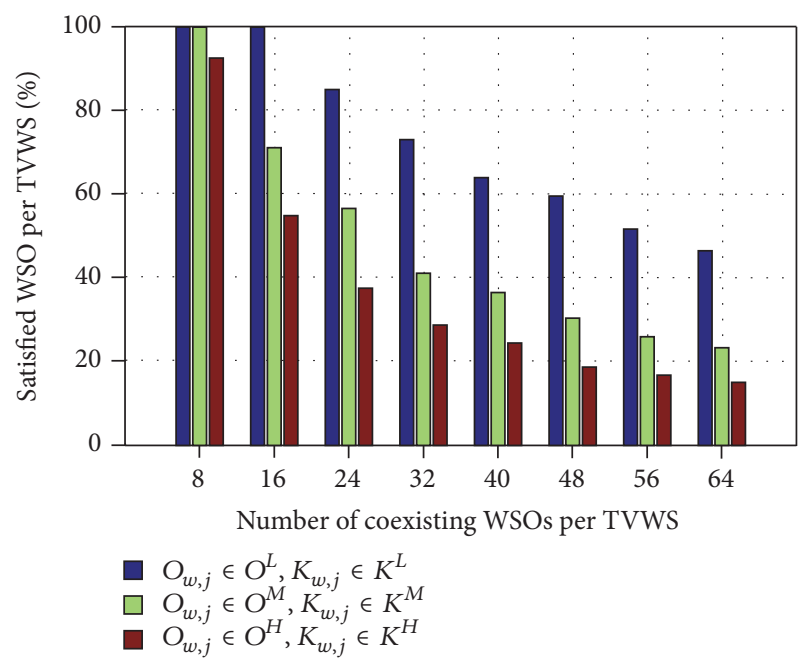

FIGURE 8: WSOs satisfied from allocation with varying WSO density in the region. The number of TV channels in the system is 4 .

channel quality, the WSOs channel demands, and the WSO density in the system, one can estimate an optimal number of WSOs that can be accommodated on the available TVWS such that the resource utilization is maximized.

6.5. Algorithm Scalability Test. The scalability of the proposed algorithm in terms of time taken to complete the allocation process is evaluated. In this experiment, the total number of coexisting WSOs registered in all the CMs in the system varies geometrically as $2^{W}$, where $W \in\{3,4,5,6,7\}$. The number of TV channels in the system increases at a constant interval of 4 as $J \in\{4,8,12, \ldots, 48\}$. The remaining simulation parameters are the same as defined in Section 6.2. The specifications of the computer system used for the scalability test are listed in Table 2. Using the above parameters, the intlinprog routine solves the proposed TVWS sharing problem. The routine uses the branch and bound method
TABLE 2: Computer system configuration.

\begin{tabular}{lcc}
\hline Symbol & Description & Quantity \\
\hline Processor & Intel quad & CPU $=3.30 \mathrm{GHz}$ \\
Onboard memory & - & $8555 \mathrm{MB}$ \\
Memory used by MATLAB & - & $1289 \mathrm{MB}$ \\
\hline
\end{tabular}

to find an optimal solution point $\mathbf{X}$. The branch and bound split the problem into subproblems, and each subproblem is expanded until a solution is found as long as its cost does not exceed the set upper bound. The exact computational complexity of any branching algorithm is hard to find as time complexity of such a branching algorithm is usually analyzed by the method of branching vector. However, it has been mentioned in [46] that when the best-first search branch and bound technique is used, the upper bound to generate an expected solution is $\sum_{i=0}^{n} T(i) \leq \sum_{i=0}^{n} n-i+1 \leq(n+1)^{2}$, where $n$ is the number of nodes visited. Thus, the complexity of such an algorithm is $\mathcal{O}\left(n^{2}\right)$.

In this experiment, we measure the simulation time taken using the MATLAB tic-toc stopwatch timer. The time recorded for the high domain channel assignment is shown in Figure 9. The result in this figure is generated using the average time required to complete allocation for the high subdomain case: that is, $O_{w, j}^{c} \in O^{H}$ and $K_{w, j}^{c} \in K^{H}$. In this study, we perform the population engineering step, as defined in Section 6.2, using the intlinprog routine of the MATLAB. The figure indicates that, for defined simulation parameters, the channel sharing process took a few hundreds of milliseconds to complete the allocation process which is quite acceptable for real-time implementation of the algorithm. Figure 9 shows that the algorithm execution time does not grow geometrically as the number of WSOs in the system increases. Rather, the algorithm has linear time allocation behavior as shown in Figure 9.

\section{Conclusion}

In this study, we investigated the channel sharing problem in a TVWS sharing domain with the objective of maximizing the resource utilization. The defined TVWS sharing problem optimizes the system throughput under a minimum fairness in allocation while constrained to satisfy the WSO channel occupancy demand on each allocated channel. To solve the defined problem, we proposed a channel allocation algorithm that shares the available TVWS among coexisting WSOs operating on incompatible network technologies. In order to improve the TVWS utilization and to accommodate as many as WSOs in the available TVWS, the proposed algorithm spatially reuse the available TV spectrum. The simulation results show that the frequency reuse property of the proposed algorithm results in comparatively higher WSO satisfaction from the allocation, better fairness in allocation, and higher system throughput gain. Moreover, the fast allocation process of the proposed algorithm makes it a promising candidate for implementation in 802.19.1 based coexistence system. The 


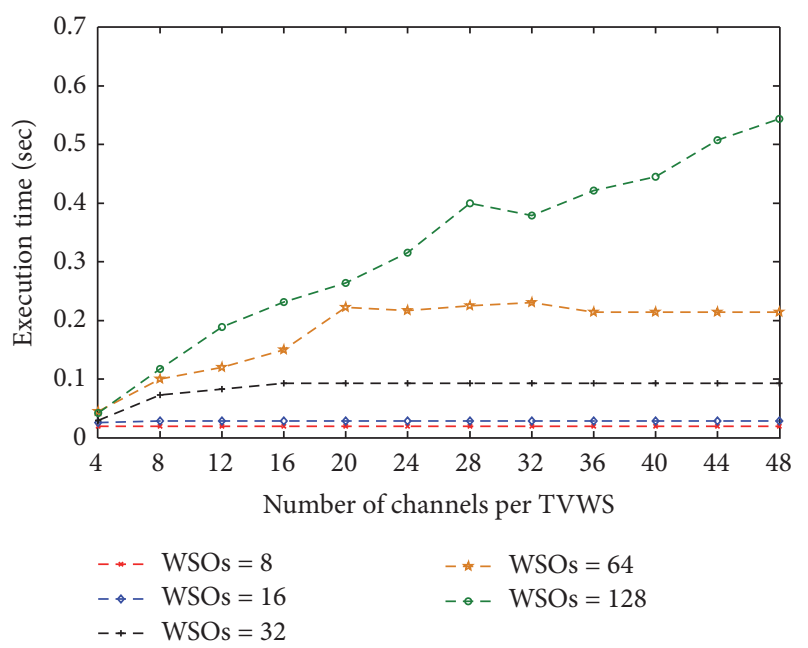

FIGURE 9: Algorithm execution time for varying number of WSOs and varying number of TV channels in the system.

proposed algorithm can be implemented by a centralized decision-making entity, that is, the master CM in the IEEE 802.19.1 system without requiring any major change in the baseline architecture of 802.19.1 TVWS sharing system.

\section{Appendix}

\section{A. Linearization Using Tangent Plane Approximation}

In this section, we apply tangent plane approximation to linearize the objective function in (2la).

Let, for some given points on the graph, $\left(q_{1}=x_{1, j}^{c}, q_{2}=\right.$ $\left.x_{2, j}^{c}\right)$ and $\mathrm{F}=\log (q+1)$, where $q=q_{1} r_{1, j}^{c} /\left(O_{1, j}^{c}+\delta_{O_{1, j}^{c} 0}\right)+$ $q_{2} r_{2, j}^{c} /\left(O_{2, j}^{c}+\delta_{O_{2, j}^{c} 0}\right)$. If $\log \left(\mathcal{U}_{c, j}+1\right)$ is differentiable at $\left(q_{1}, q_{2}\right)$, then the surface has tangent plane at $\left(q_{1}, q_{2}, \mathrm{~F}\right)$. The equation of the tangent plane at $\left(q_{1}, q_{2}, F\right)$ is given by

$$
\begin{gathered}
\frac{\partial y}{\partial x_{1, j}^{c}}\left(q_{1}, q_{2}\right)\left(x_{1, j}^{c}-q_{1}\right)+\frac{\partial y}{\partial x_{2, j}^{c}}\left(q_{1}, q_{2}\right)\left(x_{2, j}^{c}-q_{2}\right) \\
-(F-F)=0,
\end{gathered}
$$

where $y$ denotes multivariate objective function $\log \left(\mathcal{U}_{c, j}+1\right)$ and $F=\log \left(\mathcal{U}_{c, j}+1\right)$.

The tangent plane equation is rearranged as

$$
\begin{aligned}
F= & \mathrm{F}+\frac{\partial y}{\partial x_{1, j}^{c}}\left(q_{1}, q_{2}\right)\left(x_{1, j}^{c}-q_{1}\right) \\
& +\frac{\partial y}{\partial x_{2, j}^{c}}\left(q_{1}, q_{2}\right)\left(x_{2, j}^{c}-q_{2}\right),
\end{aligned}
$$

where $\partial y / \partial x_{1, j}^{c}=r_{1, j}^{c} /\left(\mathcal{U}_{c, j}+1\right)\left(O_{1, j}^{c}+\delta_{O_{1, j}^{c} 0}\right)$ denotes partial derivative of $\log$ function at $x_{1, j}^{c}$. Thus, if $\mathrm{F}$ is differentiable at $\left(q_{1}, q_{2}\right)$, then the tangent plane to the surface at $\left(q_{1}, q_{2}\right)$ provides a good approximation to $\mathrm{F}$ near $\left(q_{1}, q_{2}\right)$,

$$
\begin{aligned}
F \approx F & +\frac{\partial y}{\partial x_{1, j}^{c}}\left(q_{1}, q_{2}\right)\left(x_{1, j}^{c}-q_{1}\right) \\
& +\frac{\partial y}{\partial x_{2, j}^{c}}\left(q_{1}, q_{2}\right)\left(x_{2, j}^{c}-q_{2}\right)
\end{aligned}
$$

which is called linear approximation of $y$ near $\left(q_{1}, q_{2}\right)$.

For a general case with $\left|\mathscr{W}^{c}\right|=n$, and near to some given point, $\mathbf{q}=q_{1}=x_{1, j}^{c}, \ldots, q_{n}=x_{n, j}^{c}$, we define linear approximation of $y$ as

$$
\begin{gathered}
F \approx \mathrm{F}+\frac{\partial y}{\partial x_{1, j}^{c}}(\mathbf{q})\left(x_{1, j}^{c}-q_{1}\right)+\cdots \\
+\frac{\partial y}{\partial x_{n, j}^{c}}(\mathbf{q})\left(x_{n, j}^{c}-q_{n}\right) .
\end{gathered}
$$

\section{B. Convergence Condition for Algorithm 2}

In this section, we aim to discuss the convergence property of the algorithm in Algorithm 1. Note that our discussion here closely follows the discussion on the convergence of subgradient algorithm defined in [47]. Interested readers are referred to [47] for seeking knowledge beyond what is presented in this short discussion.

Given $\lambda^{0} \in E^{W}$ and the sequence $\left\{t_{k}\right\}$ of positive scalars, called step sizes, in Algorithm 1, define the sequence $\left\{\lambda^{k}\right\}$ as defined in Step 5(b) in Algorithm 1,

$$
\lambda^{k+1}=\max \left\{\lambda^{k}+t_{k} \nabla h\left(\lambda^{k}\right), 0\right\} .
$$

For any $\lambda$, the maximum of (24) is assumed for at least one value of the index $k$. Since (24) is piecewise linear, there then exists at least one point $\lambda^{*}$ such that $h\left(\lambda^{*}\right)=h^{*}=$ $\max P\left(\mathbf{X}, \lambda^{*}\right)$. Then, $h\left(\lambda^{k}\right)$ will converge to its optimum $h^{*}$ under the conditions,

$$
\begin{gathered}
\lim _{k \rightarrow \infty} t^{k} \longrightarrow 0 \\
\sum_{k=0}^{\infty} t_{k}=\infty
\end{gathered}
$$

For the proof of the convergence of subgradient algorithm, the interested readers are encouraged to refer to [47].

\section{Conflicts of Interest}

The authors declare that they have no conflicts of interest.

\section{Acknowledgments}

This work was supported by the National Research Foundation of Korea (NRF) funded by the Korean Government (MSIP) under Grant NRF-2015R1A2A1A05001826. 


\section{References}

[1] "Cisco Visual Networking Index: Global Mobile Data Traffic Forecast Update, 2016-2021," Tech. Rep., San Jose, Calif, USA, 2017.

[2] Wikipedia, "White spaces (Radio)," 2011, https://en.wikipedia .org/wiki/White_spaces_(radio).

[3] FCC, "Third memorandum opinion and order in the matter of unlicensed operation in the TV broadcast bands, additional spectrum for unlicensed devices below $900 \mathrm{MHz}$ and in the 3 GHz band," docket 02-380, 2012.

[4] Ofcom, "Implementing TV White Spaces," 2015.

[5] Industry Canada, "Consultation on a Policy and Technical Framework for the Use of Non-Broadcasting Applications in the Television Broadcasting Bands Below $698 \mathrm{MHz}$," 2011.

[6] T. Baykas, M. Kasslin, M. Cummings et al., "Developing a standard for TV white space coexistence: Technical challenges and solution approaches," IEEE Wireless Communications, vol. 19, no. 1, pp. 10-22, 2012.

[7] C. Ghosh, S. Roy, and D. Cavalcanti, "Coexistence challenges for heterogeneous cognitive wireless networks in TV white spaces," IEEE Wireless Communications, vol. 18, no. 4, pp. 22-31, 2011.

[8] L. Akter and B. Natarajan, "Modeling fairness in resource allocation for secondary users in a competitive cognitive radio network," in Proceedings of the 9th Annual Wireless Telecommunications Symposium, WTS 2010, Tampa, FLa, USA, April 2010.

[9] G. P. Villardi, Y. D. Alemseged, C. Sun et al., "Enabling coexistence of multiple cognitive networks in TV white space," IEEE Wireless Communications, vol. 18, no. 4, pp. 32-40, 2011.

[10] IEEE Standard 802.19.1, "TV white space coexistence methods," 2014.

[11] B. Bahrak and J.-M. J. Park, "Coexistence decision making for spectrum sharing among heterogeneous wireless systems," IEEE Transactions on Wireless Communications, vol. 13, no. 3, pp. 1298-1307, 2014.

[12] IEEE Standard 802.15.2, "Coexistence of wireless personal area networks with other wireless devices operating in unlicensed frequency bands," 2003.

[13] S. Pollin, I. Tan, B. Hodge, C. Chun, and A. Bahai, "Harmful coexistence between 802.15.4 and 802.11: A measurement-based study," in Proceedings of the 3rd International Conference on Cognitive Radio Oriented Wireless Networks and Communications, CrownCom 2008, Singapore, Singapore, May 2008.

[14] IEEE Standard 802.11af, "Wireless LAN medium access control (MAC) and physical layer (PHY) specifications-Television white spaces (TVWS) operation," 2014.

[15] IEEE Standard 802.22.1, "Standard to Enhance Harmful Interference Protection for Low-Power Licensed Devices Operating in TV Broadcast Bands," 2011.

[16] "MAC and PHY for Operation in TV White Space," std. ECMA392, 2012.

[17] F. Hessar and S. Roy, "Resource allocation techniques for cellular networks in TV white space spectrum," in Proceedings of the 2014 IEEE International Symposium on Dynamic Spectrum Access Networks, DYSPAN 2014, pp. 72-81, McLean, Va, USA, April 2014.

[18] T. Bansal, D. Li, and P. Sinha, "Opportunistic channel sharingin cognitive radio networks," IEEE Transactions on Mobile Computing, vol. 13, no. 4, pp. 852-865, 2014.

[19] R. T. Marler and J. S. Arora, "Survey of multi-objective optimization methods for engineering," Structural and Multidisciplinary Optimization, vol. 26, no. 6, pp. 369-395, 2004.
[20] K. Khalil, G. Farhadi, and A. Ito, "Coexistence management for heterogeneous networks in white spaces," in Proceedings of the 2014 International Conference on Computing, Networking and Communications, ICNC 2014, pp. 691-697, Honolulu, Hawaii, USA, February 2014.

[21] D. Zhang, Q. Liu, L. Chen, and W. Xu, "Ecology-based coexistence mechanism in heterogeneous cognitive radio networks," in Proceedings of the 58th IEEE Global Communications Conference, GLOBECOM 2015, San Diego, Calif, USA, December 2015.

[22] K. Bian, J. M. Park, and B. Gao, "Frequency reuse over a single TV white space channel," in Cognitive Radio Networks, pp. 77100, Springer, 2014.

[23] B. Gao, J.-M. J. Park, and Y. Yang, "Uplink Soft frequency reuse for self-coexistence of cognitive radio networks," IEEE Transactions on Mobile Computing, vol. 13, no. 6, pp. 1366-1378, 2014.

[24] L. M. O. Khanbary and D. P. Vidyarthi, "Reliability-based channel allocation using genetic algorithm in mobile computing," IEEE Transactions on Vehicular Technology, vol. 58, no. 8, pp. 4248-4256, 2009.

[25] A. P. Shrestha, J. Won, S.-J. Yoo, M. Seo, and H.-W. Cho, "Genetic algorithm based sensing and channel allocation in cognitive ad-hoc networks," in Proceedings of the 2016 International Conference on Information and Communication Technology Convergence, ICTC 2016, pp. 109-111, Jeju, South Korea, October 2016.

[26] Y. Jiao and I. Joe, "Energy-efficient resource allocation for heterogeneous cognitive radio network based on two-tier crossover genetic algorithm," Journal of Communications and Networks, vol. 18, no. 1, Article ID 000014, pp. 112-122, 2016.

[27] D. T. Ngo, C. Tellambura, and H. H. Nguyen, "Efficient resource allocation for OFDMA multicast systems with spectrumsharing control," IEEE Transactions on Vehicular Technology, vol. 58, no. 9, pp. 4878-4889, 2009.

[28] M. R. Sherif, I. W. Habib, M. Nagshineh, and P. Kermani, "Adaptive allocation of resources and call admission control for wireless ATM using genetic algorithms," IEEE Journal on Selected Areas in Communications, vol. 18, no. 2, pp. 268-282, 2000.

[29] M. Y. Alias, S. Chen, and L. Hanzo, "Multiple-antenna-aided OFDM employing genetic-algorithm-assisted minimum bit error rate multiuser detection," IEEE Transactions on Vehicular Technology, vol. 54, no. 5, pp. 1713-1721, 2005.

[30] F. Hessar and S. Roy, "Capacity Considerations for Secondary Networks in TV White Space," IEEE Transactions on Mobile Computing, vol. 14, no. 9, pp. 1780-1793, 2015.

[31] L. E. Li, M. Pal, and Y. R. Yang, "Proportional Fairness in MultiRate Wireless LANs," in Proceedings of the 27th Conference on Computer Communications, INFOCOM 2008, pp. 1004-1012, Phoenix, Ariz, USA, April 2008.

[32] T. Bonald, L. Massoulié, A. Proutière, and J. Virtamo, "A queueing analysis of max-min fairness, proportional fairness and balanced fairness," Queueing Systems. Theory and Applications, vol. 53, no. 1-2, pp. 65-84, 2006.

[33] L. B. Jiang and S. C. Liew, "Proportional fairness in wireless LANs and ad hoc networks," in Proceedings of the 2005 IEEE Wireless Communications and Networking Conference, WCNC 2005: Broadband Wirelss for the Masses - Ready for Take-off, pp. 1551-1556, New Orleans, La, USA, March 2005.

[34] F. Kelly, "Charging and rate control for elastic traffic," European Transactions on Telecommunications, vol. 8, no. 1, pp. 33-37, 1997. 
[35] T. Bu, L. Li, and R. Ramjee, "Generalized proportional fair scheduling in third generation wireless data networks," in Proceedings of the 25th IEEE International Conference on Computer Communications (INFOCOM '06), pp. 1-12, Barcelona, Spain, April 2006.

[36] C. Cano and D. J. Leith, "Coexistence of WiFi and LTE in unlicensed bands: a proportional fair allocation scheme," in Proceedings of the IEEE International Conference on Communication Workshop (ICCW'15), pp. 2288-2293, London, UK, June 2015.

[37] F. P. Kelly, A. K. Maulloo, and D. Tan, "Rate control for communication networks: Shadow prices, proportional fairness and stability," Journal of the Operational Research Society, vol. 49, no. 3, pp. 206-217, 1997.

[38] S.-S. Byun and C. Yoo, "Minimum DVS gateway deployment in DVS-based overlay streaming," Computer Communications, vol. 31, no. 3, pp. 537-550, 2008.

[39] L. Daniel and K. Narayanan, "Congestion Control 2: Utility, Fairness, and Optimization in Resource Allocation," Lecture Notes, 2013.

[40] W. Murray and K.-M. Ng, "An algorithm for nonlinear optimization problems with binary variables," Computational Optimization and Applications. An International Journal, vol. 47, no. 2, pp. 257-288, 2010.

[41] M. Pioro and D. Medhi, "General optimization methods for network design," in Routing, Flow, and Capacity Design in Communication and Computer Networks, pp. 178-184, Elsevier, USA, 2004.

[42] R. Jain, "Selection of techniques and metrics," in The Art of Computer Systems Performance Analysis: Techniques for Experimental Design Measurements Simulation and Modeling, pp. 3040, Wiley Computer Publishing, 1991.

[43] R. Jain, D. M. Chiu, and W. Hawe, "A quantitative measure of fairness and discrimination for resource allocation in shared computer systems," Tech. Rep., Eastern Research Lab., Dig. Equip. Corp., Hudson, Mass, USA, 1984, http://www.cse.wustl .edu/ jain/papers/ftp/fairness.pdf.

[44] "IEEE Standard for information technology, Part 22: Cognitive wireless RAN medium access control (MAC) and physical layer (PHY) specifications: Policies and procedures for operation in the TV Bands," IEEE Std 802.22-2011, 2011.

[45] T. Robert, "Data communications fundamentals," in Data Communications: An Introduction to Concepts and Design, Elsevier, USA, 2013.

[46] A. Kugel, "Proofs for the paper: Average Case Complexity of Branch-and-Bound Algorithms on Random b-ary Trees," Proofs Report, Faculty of Engineering and Computer Sciences, Ulm University, Ulm, Germany, https://www.uni-ulm.de/fileadmin/website_uni_ulm/iui.inst.190/Mitarbeiter/kuegel/proofs .pdf.

[47] M. Held, P. Wolfe, and H. P. Crowder, "Validation of subgradient optimization," Mathematical Programming, vol. 6, pp. 62-88, 1974. 


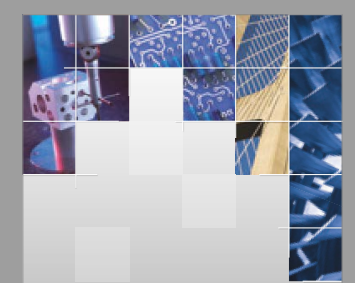

\section{Enfincering}
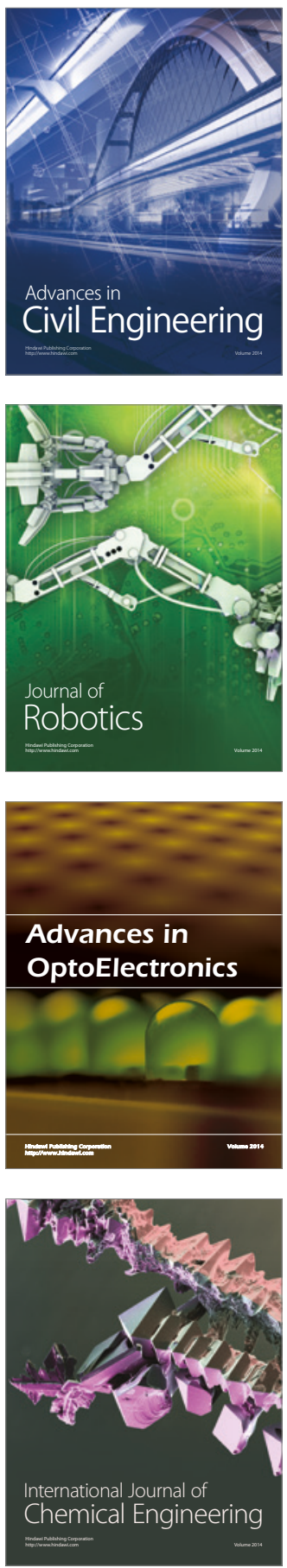

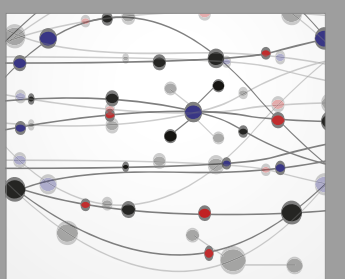

The Scientific World Journal

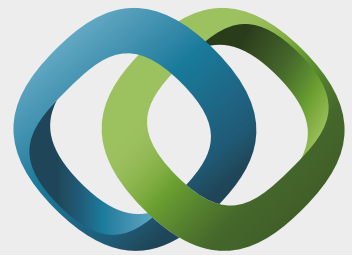

\section{Hindawi}

Submit your manuscripts at

https://www.hindawi.com
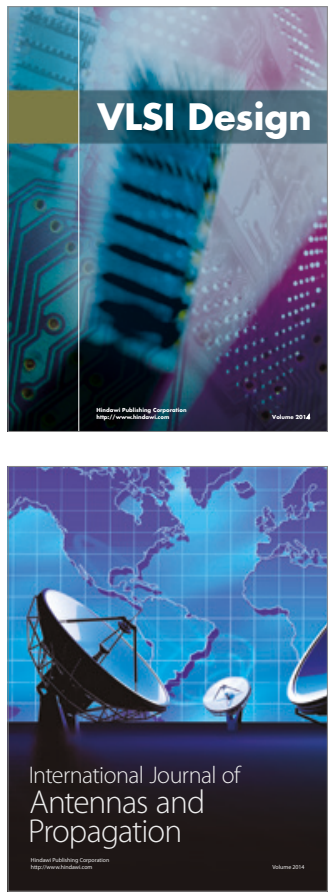

\section{Rotating}

Machinery
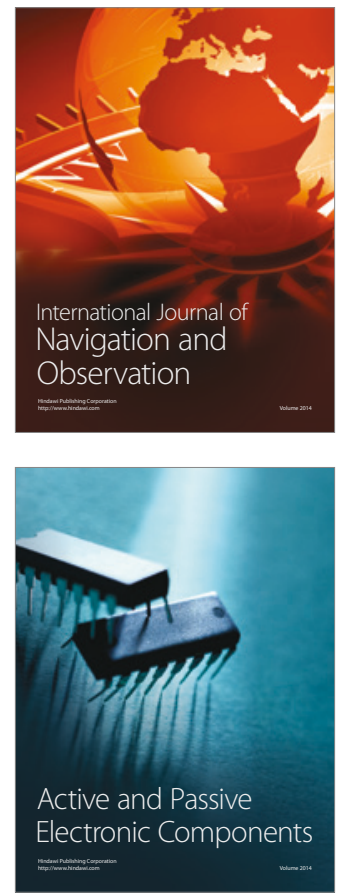
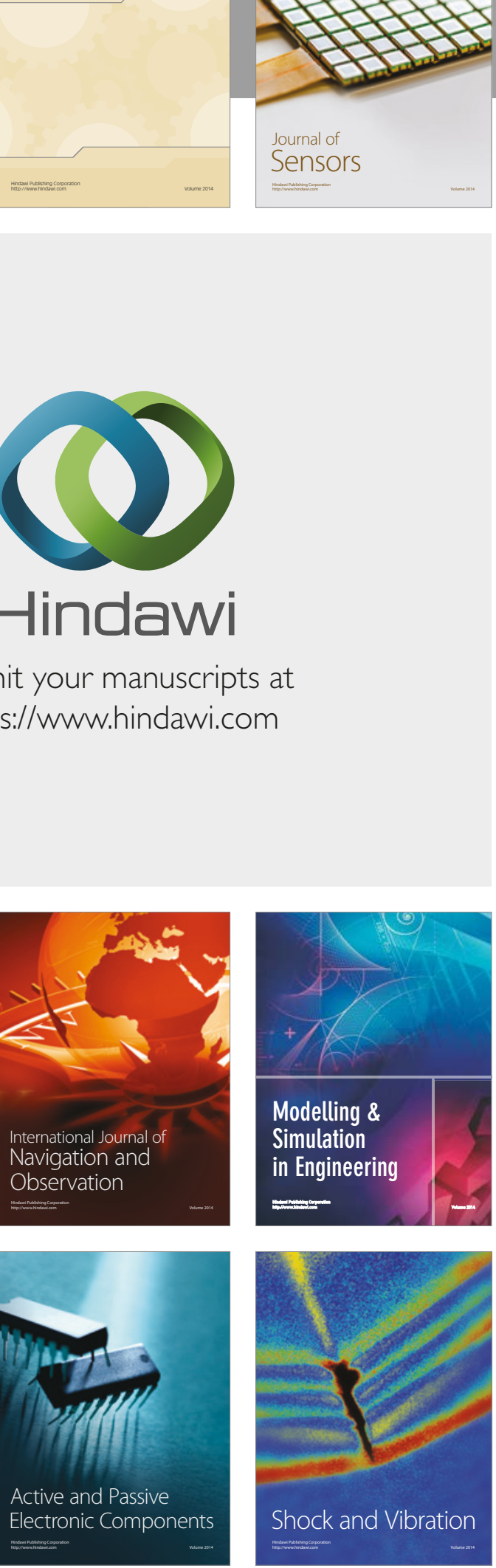
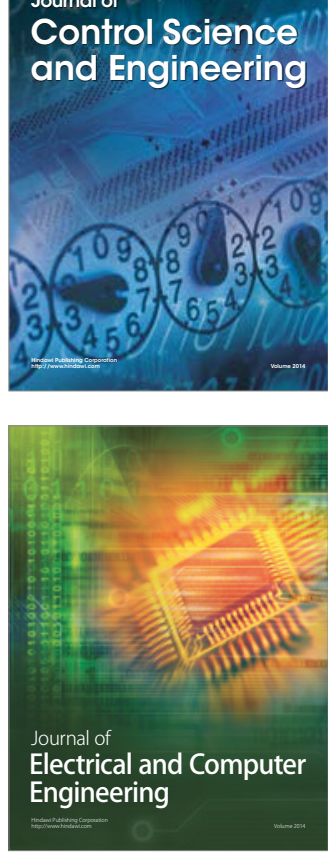

Distributed

Journal of

Control Science

and Engineering
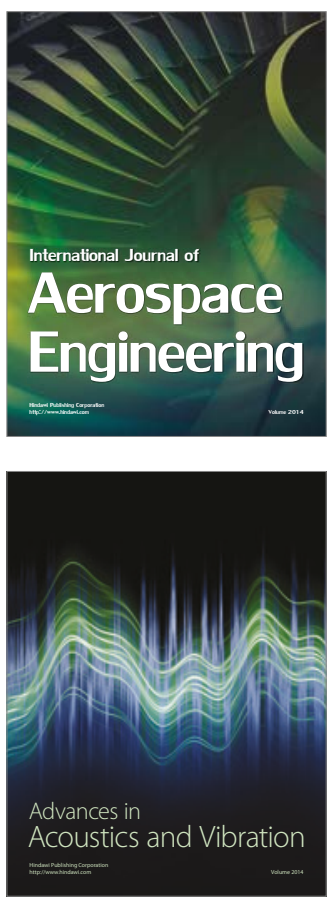

Sensor Networks 